\title{
Review Article \\ Quarkonium Production and Proposal of the New Experiments on Fixed Target at the LHC
}

\author{
A. B. Kurepin and N. S. Topilskaya \\ Institute for Nuclear Research, RAS, 60th October Anniversary Prospect 7a, Moscow 117312, Russia \\ Correspondence should be addressed to N. S. Topilskaya; topilska@inr.ru
}

Received 20 March 2015; Revised 26 May 2015; Accepted 28 June 2015

Academic Editor: Jibo He

Copyright (C) 2015 A. B. Kurepin and N. S. Topilskaya. This is an open access article distributed under the Creative Commons Attribution License, which permits unrestricted use, distribution, and reproduction in any medium, provided the original work is properly cited. The publication of this article was funded by SCOAP ${ }^{3}$.

\begin{abstract}
The brief review of the experimental data on quarkonium productions measured at the CERN SPS, at the Brookhaven Collider RHIC, and at the LHC is presented. The dissociation of quarkonium resonances produced in heavy ion collisions was suggested as a possible signal of the Quark-Gluon Plasma formation. At the CERN SPS, the anomalous suppression of the $J / \psi$ production was observed in central $\mathrm{Pb}-\mathrm{Pb}$ collisions by the NA50 collaboration. However, the effects of $J / \psi$ suppression on cold nuclear matter, feed-down production from higher charmonium states, and regeneration processes should be taken into account. If proton and ion beams at the LHC will be used with fixed targets, the energy interval between the SPS energy and the nominal RHIC energy $(200 \mathrm{GeV})$ could be investigated. The high statistics data on quarkonium productions at these energies will give the possibility of clarifying the mechanism of charmonium productions to investigate the importance of the recombination process, since the probability of recombination decreases with decreasing the energy of collisions.
\end{abstract}

\section{Introduction}

The existence of the Quark-Gluon Plasma (QGP) is predicted by lattice QCD at high temperature and large energy density. Quarkonia are important probes to study the properties of the deconfined matter. The dissociation of heavy-quark resonances by color screening in a deconfined medium was suggested by Matsui and Satz as a possible signal of the Quark-Gluon Plasma formation in ultrarelativistic heavy ion collisions [1].

Quarkonium productions have been previously studied at the CERN SPS by NA38 [2], NA50 [3-7], and NA60 $[8,9]$ experiments, at the FNAL $[10]$, and by fixed target p-A experiments at the HERA-B [11]. In 1997, the NA50 experiment has observed an "anomalous" suppression of the $J / \psi$ production in central $\mathrm{Pb}-\mathrm{Pb}$ collisions [3]. However, the cold nuclear matter (CNM) effects and feed-down production of $J / \psi$ from higher charmonium states, which affect the extraction of hot and dense matter effects, should be carefully measured. In spite of many experimental results already obtained on the $J / \psi$ production in p-A collisions, they are still not well understood, especially at small Bjorken $x_{B}$ and large Feynman $x_{F}$, where the CNM effects are expected to be large. Quarkonium productions have been studied in the Brookhaven National Laboratory at RHIC by PHENIX [12-16] and STAR experiments [17-19]. It was shown that the $J / \psi$ suppression measured by PHENIX experiment at $\sqrt{s}=200 \mathrm{GeV}$ is of the same order as the suppression at the SPS energies for $\mathrm{Pb}-\mathrm{Pb}$ collisions $[13,20]$. In order to get better agreement between theoretical description of the RHIC and the SPS experimental results the models that include the regeneration of $J / \psi$ were developed [21-24]. The quarkonium productions have been measured at significantly higher energies at the LHC by ALICE [25, 26], CMS [27, 28], ATLAS $[29,30]$, and LHCb $[31,32]$ experiments. At the LHC energies the contribution of B-decay should be taken into account $[27,29,31-33]$. The high statistics measurements at the LHC could investigate the properties of matter at high energy density and temperature. However, the quarkonium study at energies below the LHC energy is also very important 
in order to investigate the mechanism of production, the medium effects, and conditions for the Quark-Gluon Plasma formation. If it would be possible to use the proton and ion beams at the LHC with fixed targets, the data in the energy interval between maximum energy of the SPS $(\sqrt{s} \sim$ $29 \mathrm{GeV})$ and the nominal RHIC energy $(\sqrt{s}=200 \mathrm{GeV})$ in p$\mathrm{A}$ and A-A collisions could be obtained. For $0.45 \mathrm{TeV}$ proton beam, the energy in N-N centre-of-mass system is $\sqrt{s_{\mathrm{NN}}}$ $=29.1 \mathrm{GeV}$, for $7 \mathrm{TeV}$ proton beam $\sqrt{s_{\mathrm{NN}}}=114.6 \mathrm{GeV}$ and for $2.75 \mathrm{TeV} \mathrm{Pb}$ beam $\sqrt{s_{\mathrm{NN}}}=71.8 \mathrm{GeV}$. The high statistics data on quarkonium production at these energies will give the possibility of clarifying the mechanism of charmonium production to investigate the importance of recombination process, since the probability of recombination decreases with decreasing the energy of collisions.

\section{Charmonium Production at the CERN SPS}

The productions of $J / \psi$ and $\psi(2 S)$ have been studied at the CERN SPS by the NA50 experiment. Data have been taken for $\mathrm{Pb}-\mathrm{Pb}$ collisions at $158 \mathrm{GeV}$ per nucleon $[3,7]$ and for p-A collisions at $450[4,5]$ and $400 \mathrm{GeV}[6]$. The nuclear suppression of the $J / \psi$ production in proton-nucleus reactions and an anomalous suppression in central lead-lead collisions were observed $[3,7]$. In the NA60 experiment, the $J / \psi$ production was measured in In-In collisions at $158 \mathrm{GeV}$ per nucleon [8] and in p-A collisions at 400 and $158 \mathrm{GeV}$ [9]. The NA60 results in p-A collisions at $400 \mathrm{GeV}$ confirm the NA50 values of absorption cross section at the same energy. On the other hand, the NA60 $158 \mathrm{GeV}$ p-A data give higher values of absorption. Rather strong energy dependence of the absorption cross section was observed. However, the older NA3 J/ $\psi$ results at $200 \mathrm{GeV}$ [34] are in partial contradiction with these observations and give values of absorption cross section similar to those obtained at the higher energy. This difference has not yet been satisfactorily explained by theoretical models. Therefore, it is important to measure the charmonium production cross sections in $\mathrm{p}-\mathrm{p}$, $\mathrm{p}-\mathrm{A}$, and A-A collisions at the same energy and in the same kinematic domain.

The heavy-quark pair can interact with the nuclear matter and eventually break up. It is the effect of nuclear absorption. The cold nuclear matter (CNM) effect in suppression of the $J / \psi$ production includes not only nuclear absorption, but also the shadowing effect, that is, the modification of the parton distribution function in nuclear matter compared to the free nucleon. If shadowing is taken into account, the amount of the anomalous $J / \psi$ suppression is reduced. Also, it is necessary to consider the energy loss of partons in nuclear matter. The charmonium can also interact with surrounding comovers and lose energy or even break up.

For In-In collisions at $158 \mathrm{GeV}$ per nucleon after adjustment for the effects of cold nuclear matter, the additional suppression becomes quite small. But the value of the anomalous suppression in the most central $\mathrm{Pb}-\mathrm{Pb}$ collisions is of the order of $20-30 \%$. So, to extract the effects of hot and dense nuclear matter in the $J / \psi$ production at the SPS energies, the CNM effects and feed-down production from higher charmonium states must be taken into account.

\section{Charmonium Production at the Relativistic Heavy Ion Collider RHIC}

At the RHIC, the production of $J / \psi$ was measured in p-p, d-Au, $\mathrm{Au}-\mathrm{Au}$, and $\mathrm{Cu}-\mathrm{Cu}$ collisions by the PHENIX experiment at $\sqrt{s_{\mathrm{NN}}}=200 \mathrm{GeV}$ energy in the N-N centreof-mass [12-16]. To quantify the suppression of the $J / \psi$ production in heavy ion collisions, the nuclear modification factor $R_{\mathrm{AA}}$ was introduced:

$$
R_{\mathrm{AA}} \equiv \frac{d N_{\mathrm{AA}} / d y}{\left(d N_{\mathrm{pp}} / d y \cdot\left\langle N_{\text {coll }}\right\rangle\right)},
$$

which is the ratio of the $J / \psi$ yield in A-A collisions, normalized to the number of binary collisions, $N_{\text {coll }}$, to the $J / \psi$ yield in p-p collisions. The nuclear modification factor has been measured as a function of several parameters: the centrality of collisions, the multiplicity, the transverse momentum, and the number of participants $\left(N_{\text {part }}\right)$.

The suppression of the $\mathrm{J} / \psi$ production in $\mathrm{Au}-\mathrm{Au}$ collisions is considerably stronger at forward rapidity range $1.2<|y|<2.2$ than at midrapidity $|y|<0.35$. The PHENIX data for $\mathrm{Au}-\mathrm{Au}$ and d-Au collisions were analyzed simultaneously for estimation of the CNM contribution. The nuclear modification factor for cold nuclear matter $R_{\mathrm{AA}}(\mathrm{CNM})$ was obtained in $\mathrm{Au}-\mathrm{Au}$ collisions at the measured rapidity ranges [20]. The ratio $R_{\mathrm{AA}} / R_{\mathrm{AA}}(\mathrm{CNM})$ estimates the value of additional suppression of the $J / \psi$ production in the hot and dense nuclear matter produced in relativistic heavy ions collisions. The $R_{\mathrm{AA}} / R_{\mathrm{AA}}(\mathrm{CNM})$ ratio at $\sqrt{s_{\mathrm{NN}}}=200 \mathrm{GeV}$ is similar to the forward rapidity and midrapidity and reaches about $50 \%$ for the most central events.

Comparison of the $R_{\mathrm{AA}} / R_{\mathrm{AA}}(\mathrm{CNM})$ ratio estimated for NA50, NA60, and PHENIX data versus $d N / d \eta$ at pseudorapidity $\eta=0$, which is proportional to the energy density, shows that at RHIC energy anomalous suppression of $J / \psi$ for colliding nuclei is the same as at SPS energy [20] (Figure 1).

The nuclear modification factor for cold matter $R_{\mathrm{AA}}(\mathrm{CNM})$ was obtained under the assumption that in $\mathrm{p}-\mathrm{p}, \mathrm{p}-\mathrm{A}$, and $\mathrm{d}-\mathrm{Au}$ collisions at SPS and RHIC energies the hot and dense nuclear matter is not formed. There are some indications that the hot matter effects could present in $\mathrm{d}-\mathrm{Au}$ collisions at the RHIC energies [35]. These effects may contribute to $\mathrm{p}-\mathrm{p}$ and $\mathrm{p}-\mathrm{A}$ collisions at higher energies.

The production of $J / \psi$ in $\mathrm{p}-\mathrm{p}, \mathrm{Au}-\mathrm{Au}$, and $\mathrm{Cu}-\mathrm{Cu}$ collisions was measured also by STAR experiment at the RHIC at midrapidity $|y|<1$ for low $p_{T}<5 \mathrm{GeV} / \mathrm{c}$ [17] and for high $p_{T}>5 \mathrm{GeV} / \mathrm{c}[18,19]$. The value of $R_{\mathrm{AA}}$ in $\mathrm{Au}-\mathrm{Au}$ collisions shows strong suppression at low transverse momentum but the suppression decreases significantly with increasing momentum. The $J / \psi$ suppression in $\mathrm{Au}-$ $\mathrm{Au}$ collisions at low $p_{T}<5 \mathrm{GeV} / \mathrm{c}$ and at midrapidity is compatible to that measured by PHENIX. It was found that the results are consistent with models that include color screening and regeneration $[36,37]$. The data from PHENIX show a significant suppression in midcentral and central $\mathrm{Cu}-\mathrm{Cu}$ collisions [15]. The STAR $\mathrm{Cu}-\mathrm{Cu}$ data [19] exhibit no suppression, but the precision is limited by the available statistics. 


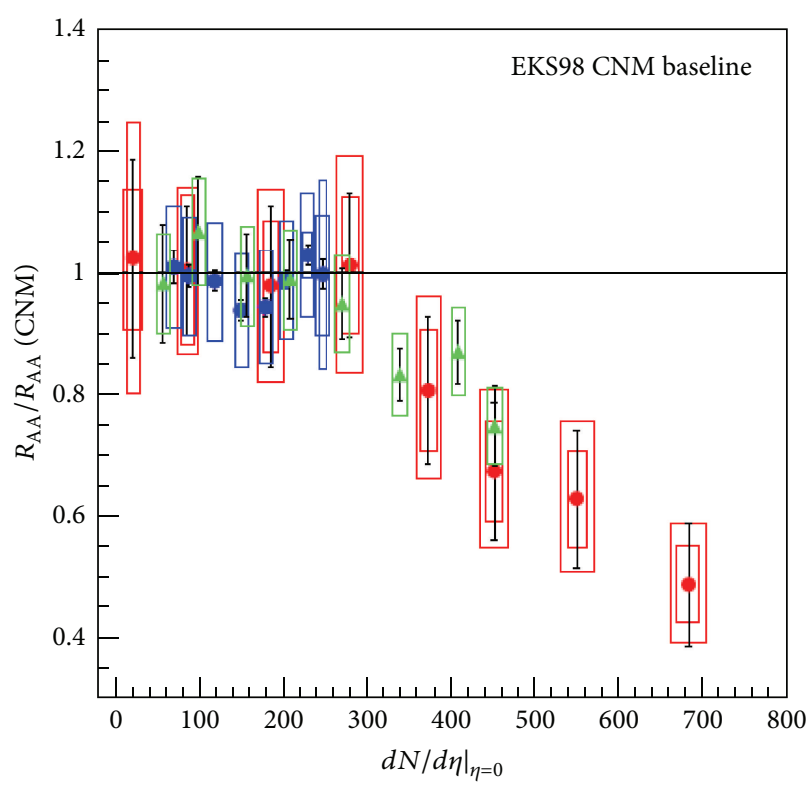

- PHENIX Au + Au $y=0 \quad$ Narrow boxes: correlated system - NA60 ln-ln

A NA50 Pb-Pb

FIGURE 1: Comparison of the $R_{\mathrm{AA}} / R_{\mathrm{AA}}(\mathrm{CNM})$ ratio obtained at the SPS and PHENIX versus $d N / d \eta$ at $\eta=0$.

The production of $J / \psi$ in asymmetric $\mathrm{Cu}-\mathrm{Au}$ heavy ion collisions was measured also at the RHIC at $\sqrt{s_{\mathrm{NN}}}=200 \mathrm{GeV}$ for both forward (Cu-going direction) and backward (Augoing direction) rapidity [38]. The suppression in the Augoing direction is found to be consistent with the suppression measured in $\mathrm{Au}-\mathrm{Au}$ collisions. In the $\mathrm{Cu}$-going direction, the $J / \psi$ suppression is stronger. The difference may be due to the CNM effects which are different at forward and backward rapidity.

Recently at the RHIC, the $J / \psi$ production in U-U collisions was measured at $\sqrt{s_{\mathrm{NN}}}=193 \mathrm{GeV}[39,40]$. The nuclear modification factor for $J / \psi$ in $U-U$ collisions for forward rapidity is very close to the $\mathrm{Au}$-Au data with a hint of a slightly weaker suppression in central U-U collisions.

The RHIC experimental data could be described by theoretical models based on the regeneration of $J / \psi$. Additional $J / \psi$ mesons are expected to be produced from deconfined charm quarks by kinetic recombination in the QGP [21, 22, $36,37]$ or by statistical hadronization at the phase boundary $[23,24]$.

Bottomonium productions were also studied at the RHIC in p-p, d-Au, and Au-Au collisions [16, 39]. The suppression of total $\Upsilon(1 S+2 S+3 S)$ yield measured by PHENIX is consistent with measurements made by STAR. The regeneration of bottomonia is expected to be small as compared to the $J / \psi$. But the energy resolution at RHIC was insufficient to separate the contributions of individual states.

The nuclear modification factor $R_{\mathrm{AA}}$ for the $J / \psi$ production measured in Au-Au collisions at lower energies 62.4 and $39 \mathrm{GeV}$ reveals approximately the same $J / \psi$ suppression as at $200 \mathrm{GeV}$ but has large statistical errors due to low luminosity and large systematical errors because of lack of p-p collisions at the same energies [41]. At the RHIC, the luminosity strongly decreases with decreasing the energy of collisions. The investigations at lower energy with high statistics are very important for understanding the mechanism of charmonium production, for investigation the contribution of cold and hot nuclear matter effects. In addition, the contribution of recombination process decreases with decreasing the energy of collision.

The fixed target experiment at high luminosity LHC beams could provide measurements for $\mathrm{p}-\mathrm{p}, \mathrm{p}-\mathrm{A}$, and $\mathrm{A}$ A collisions with the same equipment for different nuclei targets. The energy of collisions would be lower than the nominal RHIC energy $(200 \mathrm{GeV})$. The charmonium production could be investigated with high statistics and low systematic errors and may provide reference from $\mathrm{p}$-p data at these energies for RHIC.

\section{Quarkonium Production at the CERN Large Hadron Collider LHC}

At the LHC at CERN quarkonium productions were measured at the energy more than ten times higher than at RHIC. Five quarkonium states from two families are under study, charmonia: $J / \psi$ and $\psi(2 S)$ and bottomonia: $\Upsilon(1 S), \Upsilon(2 S)$, and $\Upsilon(3 S)$. In four experiments: ALICE [25, 26, 42-46], CMS [27, 28, 47-49], ATLAS [29, 30], and LHCb [31, 32, 50, 51], the quarkonium productions were measured in different energy, rapidity, and transverse momentum ranges. In all experiments $\mathrm{p}-\mathrm{p}, \mathrm{p}-\mathrm{Pb}$, and $\mathrm{Pb}-\mathrm{Pb}$ collisions were measured, except for $\mathrm{LHCb}$ where there was no $\mathrm{Pb}-\mathrm{Pb}$ program.

The quarkonium production in $\mathrm{p}$ - $\mathrm{p}$ collisions at $\sqrt{s_{\mathrm{NN}}}=$ $2.76,7$, and $8 \mathrm{TeV}$ is useful for investigating the production mechanism, for comparison with different QCD model calculations and as a reference for understanding any additional effects in $\mathrm{p}-\mathrm{A}$ and $\mathrm{A}-\mathrm{A}$ collisions. The study of quarkonium production in $\mathrm{p}-\mathrm{Pb}$ collisions at $\sqrt{s_{\mathrm{NN}}}=5.02 \mathrm{TeV}$ is important to distinguish the effects of Quark-Gluon Plasma from cold nuclear matter and to provide input to understanding of nucleus-nucleus collisions. The information about properties of hot and dense medium created in the collisions could be obtained from the measurements of $\mathrm{Pb}-\mathrm{Pb}$ collisions at $\sqrt{s_{\mathrm{NN}}}=2.76 \mathrm{TeV}$.

4.1. Charmonium Production at the LHC. At the LHC the charmonium $J / \psi$ and $\psi(2 S)$ productions were measured in p-p collisions at $2.76,7$, and $8 \mathrm{TeV}$ and in $\mathrm{Pb}-\mathrm{Pb}$ collisions at $2.76 \mathrm{TeV}$. Measurement of the $J / \psi$ production in $\mathrm{p}-\mathrm{p}$ collision at the same energy as in $\mathrm{Pb}-\mathrm{Pb}$ collision provides the reference for extracting the nuclear modification factor $R_{\mathrm{AA}}$. There is a good agreement for $\mathrm{p}$ - $\mathrm{p}$ collisions between the data obtained by ALICE [25], CMS [27], ATLAS [29], and $\mathrm{LHCb}$ [31] experiments in the same kinematic domains [52]. Comparison of charmonium $J / \psi$ and $\psi(2 S)$ production cross sections at forward rapidity in p-p collisions at $7 \mathrm{TeV}$ [42], obtained by ALICE and LHCb experiments, is shown in Figure 2.

The mechanism of charmonium production was investigated by measuring the cross sections in $\mathrm{p}-\mathrm{p}$ collisions 


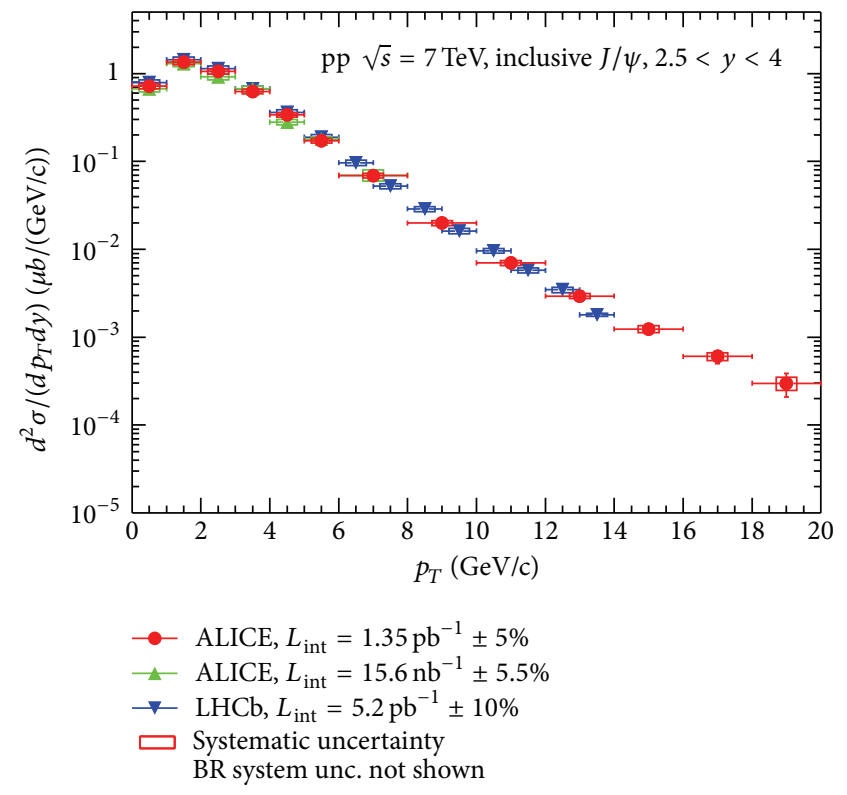

(a)

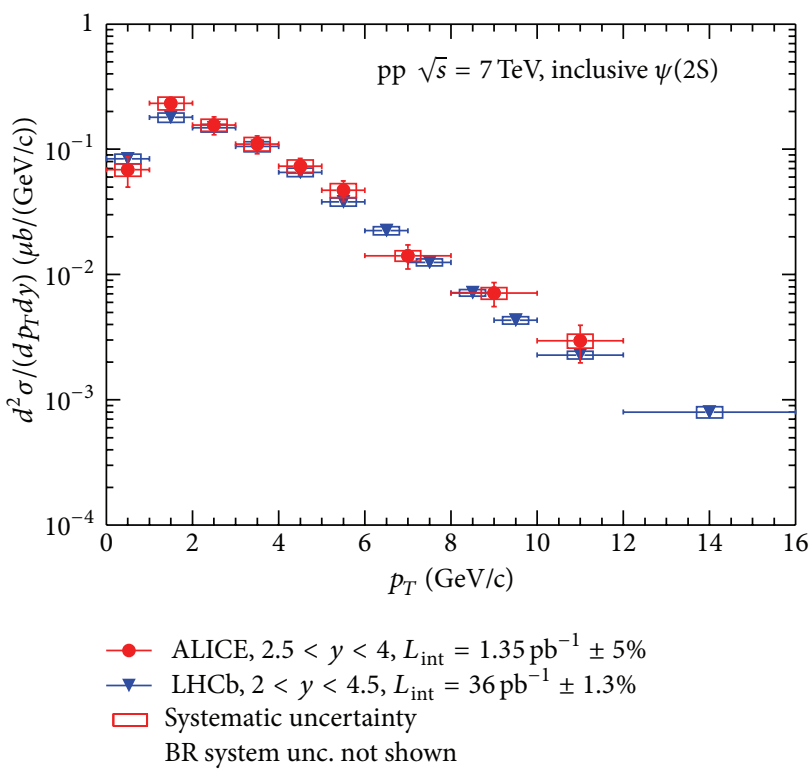

(b)

FIgURE 2: Comparison of charmonium production cross sections in p-p collisions at $7 \mathrm{TeV}$ versus transverse momentum for ALICE and $\mathrm{LHCb}$ data for $J / \psi(\mathrm{a})$ and $\psi(2 \mathrm{~S})(\mathrm{b})$.

as functions of energy, transverse momentum, and rapidity. Cross sections are compared to the theoretical models [42]. Unfortunately, none of the models, including NLO QCD, are able to describe simultaneously different aspects of quarkonium production. By increasing the energy of collisions, the mean transverse momentum and production cross section of $J / \psi$ become larger. For forward rapidity, the cross section is smaller than at midrapidity. The contribution of Bdecay to $J / \psi$ production cross section was measured. This contribution depends on rapidity, increases with growing of $J / \psi$ transverse momentum, and is of the order of $10 \%$ for transverse momentum about $1.5 \mathrm{GeV} / \mathrm{c}[27,29,33]$.

ALICE [26, 43], CMS [28], and ATLAS [30] experiments have measured charmonium productions in $\mathrm{Pb}-\mathrm{Pb}$ collisions at $\sqrt{s_{\mathrm{NN}}}=2.76 \mathrm{TeV}$. In the ALICE experiment, $J / \psi$ mesons were detected in rapidity range $|y|<0.9$ (for $J / \psi$ decay into two electrons) and $2.5<y<4$ (for muon channel) with transverse momentum values from about zero up to $8 \mathrm{GeV} / \mathrm{c}$. In ATLAS and CMS experiments, the $J / \psi$ production was measured in the rapidity range $|y|<2.4$, but the range of transverse momentum values depends on the rapidity. In ATLAS experiment, only the data for $J / \psi$ mesons production with large transverse momentum $p_{T}>6.5 \mathrm{GeV} / \mathrm{c}$ were obtained. The comparison of PHENIX and ALICE nuclear modification factors $R_{\mathrm{AA}}$ for inclusive $J / \psi$ production $[13,26]$ is shown in Figure 3.

Smaller suppression in the ALICE measurements of the inclusive $J / \psi$ production in $\mathrm{Pb}-\mathrm{Pb}$ collisions at $\sqrt{s_{\mathrm{NN}}}=$ $2.76 \mathrm{TeV}[26,43]$ compared to PHENIX results in $\mathrm{Au}-\mathrm{Au}$ collisions at $\sqrt{s_{\mathrm{NN}}}=0.2 \mathrm{TeV}$ [13] was found. These measurements are compatible with a regeneration mechanism. This additional hot nuclear matter effect works in an opposite direction to the suppression by color screening. At the LHC energy, the charm quark density produced in the collisions is larger than at SPS and RHIC energies. The probability of $c \bar{c}$ recombination increases with increasing the energy of collision and at the LHC energies may become dominant. In particular, this mechanism predicts an increase of $R_{\mathrm{AA}}$ from forward rapidity to midrapidity, where the density of charm quarks is higher. Moreover, in order to recombine, two charm quarks need to be close enough in phase space, so the effect will be larger at low $p_{T}$ of $J / \psi$ in agreement with experimental data $[36,37]$.

At the LHC, charmonium productions were measured in $\mathrm{p}-\mathrm{Pb}$ collisions at the energy $\sqrt{s_{\mathrm{NN}}}=5.02 \mathrm{TeV}$ by ALICE $[44,45]$ and LHCb $[50]$ experiments. The inclusive $J / \psi$ production has been studied by ALICE [44]. The measurement is performed down to zero transverse momentum in the center of mass rapidity domains $2.03<y<3.53$ and $-4.46<y<-2.96$ in muon channel. In p-Pb collisions, the $J / \psi$ production was also measured in electron channel in rapidity domain $-1.37<y<0.43$ [44]. Since p-p collisions data at $\sqrt{s_{\mathrm{NN}}}=5.02 \mathrm{TeV}$ for determination of $R_{\mathrm{pPb}}$ are not available, the reference $\sigma_{\mathrm{pp}}(J / \psi)$ has been obtained by an interpolation procedure, based on $\mathrm{p}-\mathrm{p}$ collisions results at $\sqrt{s_{\mathrm{NN}}}=2.76 \mathrm{TeV}$ and $7 \mathrm{TeV}$ obtained by the ALICE experiment. At forward rapidity, corresponding to the proton beam direction, a suppression of the $J / \psi$ yield with respect to binary-scaled p-p collisions is observed, but in the backward region no suppression is found. The experimental results and comparison with theoretical predictions [44] are shown in Figure 4.

The Color Glass Condensate (CGC) model [53] could not describe the data. Theoretical calculations based on 


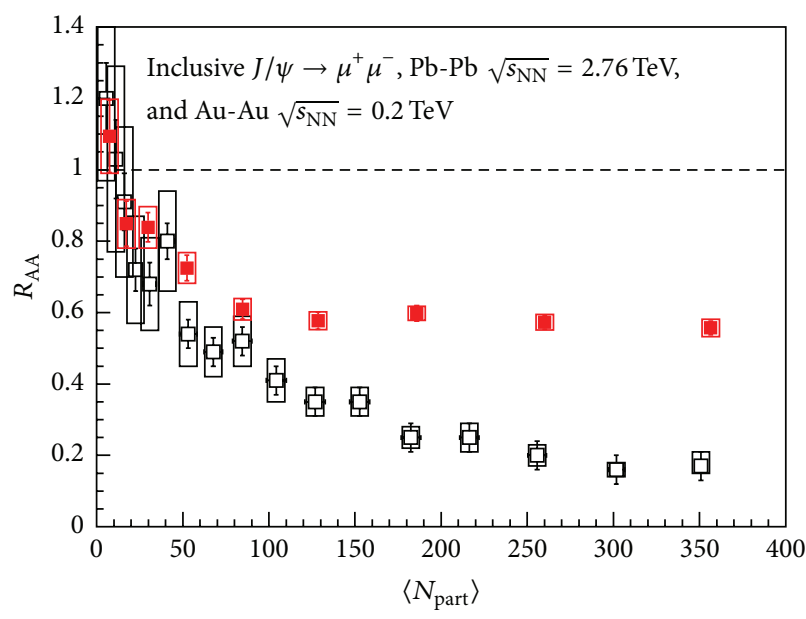

n ALICE (PLB 734 (2014) 314), $2.5<y<4,0<p_{T}<8 \mathrm{GeV} / \mathrm{c}$ Global system $= \pm 15 \%$

$\square$ PHENIX (PRC 84 (2011) 054912), $1.2<|y|<2.2, p_{T}>0 \mathrm{GeV} / \mathrm{c}$ Global system $= \pm 9.2 \%$

(a)

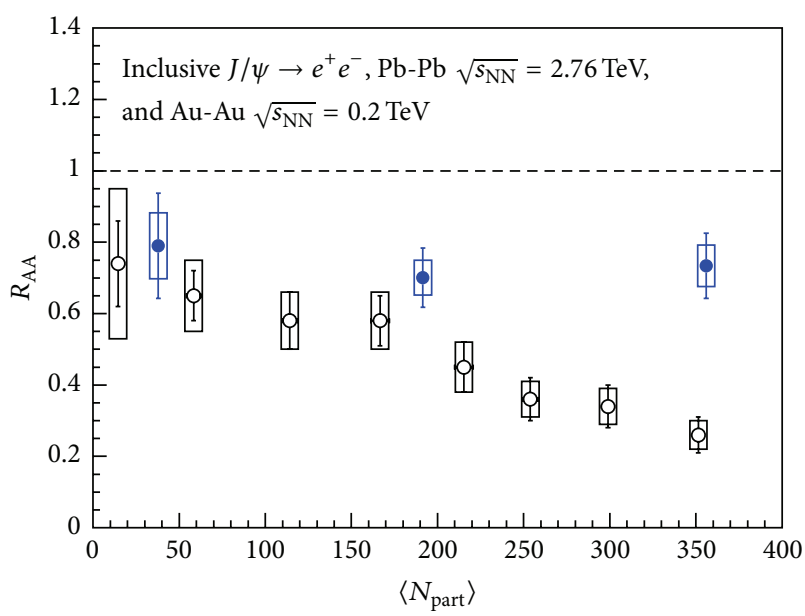

- ALICE (PLB 734 (2014) 314), $|y|<0.8, p_{T}>0 \mathrm{GeV} / \mathrm{c}$ Global system $= \pm 13 \%$

○ PHENIX (PRC 84 (2011) 054912), $|y|<0.35, p_{T}>0 \mathrm{GeV} / \mathrm{c}$ Global system $= \pm 12 \%$

(b)

FIgURE 3: Comparison of $R_{\mathrm{AA}}$ results, obtained by PHENIX and ALICE experiments. The two panels show the data at forward rapidity (a) and midrapidity (b).

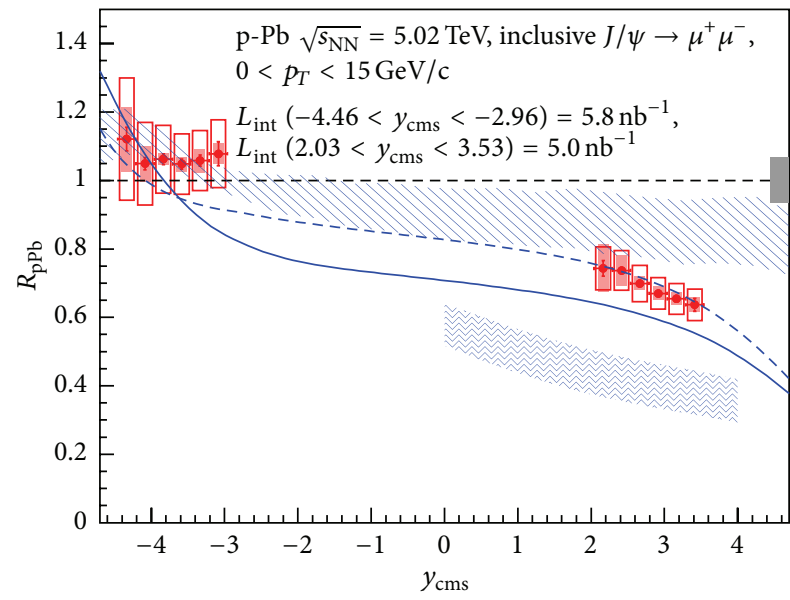

EPS09 NLO (Vogt)

CGC (Fujii and Watanable)

- - ELoss, $q_{0}=0.075 \mathrm{GeV}^{2} / \mathrm{fm}$ (Arleo and Peigné)

- EPS09 NLO + ELoss, $q_{0}=0.055 \mathrm{GeV}^{2} / \mathrm{fm}$ (Arleo and Peigné)

FIGURE 4: Comparison of ALICE $R_{\mathrm{pPb}}$ results for inclusive $J / \psi$ with theoretical predictions.

nuclear shadowing [54] as well as on the models including, in addition, a contribution from partonic energy loss $[55,56]$ are in better agreement with the experimental results (Figure 4).

The ALICE results are in agreement with results for inclusive $J / \psi$ mesons presented by LHCb collaboration [57]. Nuclear modification factors are determined separately for prompt $J / \psi$ mesons and for $J / \psi$ from B-hadron decays by
LHCb experiment. The suppression of prompt $J / \psi$ mesons in $\mathrm{p}-\mathrm{Pb}$ collisions with respect to $\mathrm{p}-\mathrm{p}$ collisions at large rapidity is observed, while the production from B-hadron decays is less suppressed.

The $\psi(2 \mathrm{~S})$ production was measured in $\mathrm{p}-\mathrm{Pb}$ collisions by ALICE [45]. The nuclear modification factor for inclusive $\psi(2 S)$ is evaluated. A significantly larger suppression of the $\psi(2 S)$ compared to the inclusive $J / \psi$ was obtained. Theoretical models predictions, which include parton shadowing and coherent energy loss mechanism, reproduce $J / \psi$ suppression but could not describe $\psi(2 S)$ data [56]. The models underestimate the $\psi(2 \mathrm{~S})$ suppression. A comparison with theoretical predictions is shown in Figure 5. Additional effects should be considered for interpretation of the results.

4.2. Bottomonium Production at the LHC. In $\mathrm{p}-\mathrm{p}$ collisions at $\sqrt{s_{\mathrm{NN}}}=7 \mathrm{TeV}$, the inclusive production cross sections of $\Upsilon(1 S)$ and $\Upsilon(2 S)$ have been measured as a function of $p_{T}$ and rapidity by ALICE [42]. There is a good agreement of ALICE data with measurements from LHCb experiment $[31,58]$ in the similar $p_{T}$ and rapidity ranges. The data are complemented to CMS measurements at midrapidity $[47,59]$. The results could be described by NLO NRQCD calculations.

The CMS Collaboration at the LHC has observed the sequential suppression of $\Upsilon(1 S), \Upsilon(2 S)$, and $\Upsilon(3 S)$ bottomonium states in $\mathrm{Pb}-\mathrm{Pb}$ collisions at $\sqrt{s_{\mathrm{NN}}}=2.76 \mathrm{TeV}[48$, 49]. The $\Upsilon(1 S)$ yield is suppressed by approximately a factor of two with respect to the expectation from $\mathrm{p}-\mathrm{p}$ collisions obtained by scaling with the number of binary nucleonnucleon collisions. The $\Upsilon(2 S)$ and the $\Upsilon(3 S)$ are strongly suppressed. Due to the lower production cross section of 


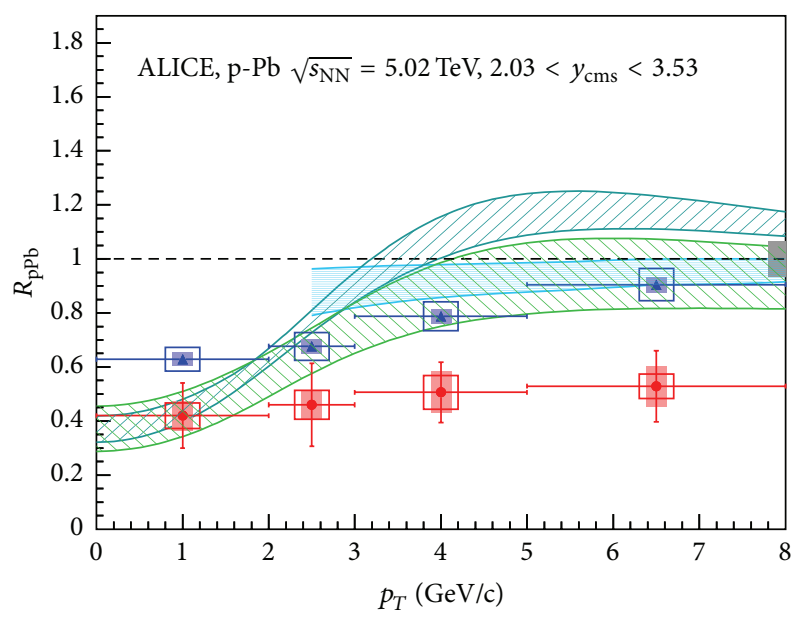

EPS09 NLO (Vogt)

ELoss with $q_{0}=0.075 \mathrm{GeV}^{2} / \mathrm{fm}$ (Arleo and Peigné)

1 EPS09 NLO + ELoss with $q_{0}=0.055 \mathrm{GeV}^{2} / \mathrm{fm}$ (Arleo and Peigné)

$\triangle \quad J / \psi$

- $\psi(2 S)$

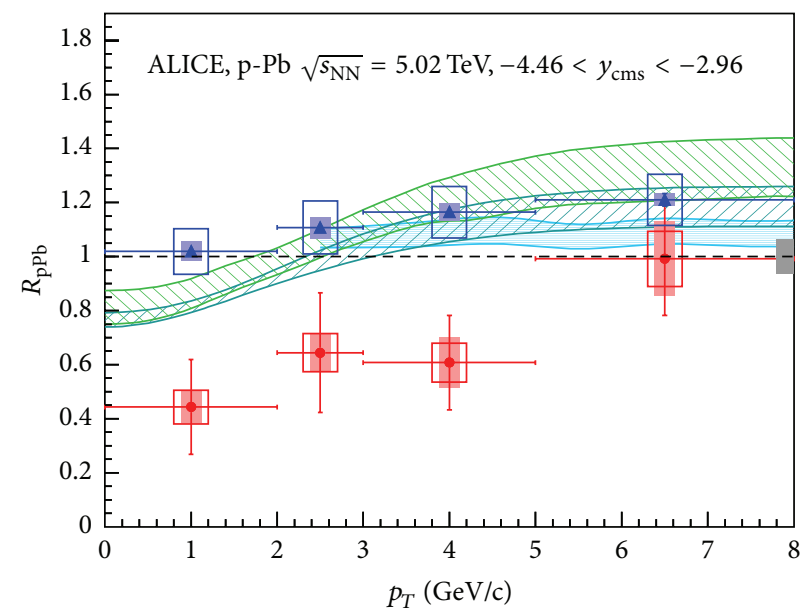

EPS09 NLO (Vogt)

ELoss with $q_{0}=0.075 \mathrm{GeV}^{2} / \mathrm{fm}$ (Arleo and Peigné)

EPS09 NLO + ELoss with $q_{0}=0.055 \mathrm{GeV}^{2} / \mathrm{fm}$ (Arleo and Peigné)

$\triangle \quad J / \psi$

- $\psi(2 S)$

FIGURE 5: Comparison of ALICE $R_{\mathrm{pPb}}$ results for $J / \psi$ and $\psi(2 S)$ at forward (a) and backward (b) rapidity with theoretical predictions.

$b \bar{b}$ pairs compared to $c \bar{c}$ pairs, the regeneration of $\Upsilon(1 S)$ is expected to be smaller than that of $J / \psi[60]$. On the other hand, the CNM effects can also modify the production of bottomonia in nucleus-nucleus collisions.

The inclusive $\mathrm{Y}(\mathrm{S} \mathrm{S})$ production in $\mathrm{Pb}-\mathrm{Pb}$ collisions was measured by ALICE at forward rapidity in muon channel down to zero transverse momentum [61]. A strong suppression was observed with respect to $\mathrm{p}-\mathrm{p}$ collisions scaled by the number of nucleon-nucleon collisions. The ALICE results are compared with CMS data $(|y|<2.4)$ [48]. The observed suppression is stronger at forward rapidity than at midrapidity. The transport model [60] predicts a nearly constant $R_{\mathrm{AA}}$ as a function of rapidity and could not reproduce ALICE and CMS data. The transport model includes both suppression and regeneration effects. CNM effects were estimated by using the effective absorption cross section. The transport model clearly underestimates the observed suppression, but the shape of the centrality dependence is quite well reproduced. Another transport model [62] also includes suppression and small regeneration component and CNM effect with shadowing. The model reproduces the CMS data but underestimates the ALICE data at forward rapidity. Both transport models $[60,62]$ predict stronger suppression for central events in agreement with centrality dependence of ALICE data. The comparison of the data with transport model calculations [60] is shown in Figure 6.

The inclusive $\Upsilon(1 S)$ and $\Upsilon(2 S)$ productions were measured also by ALICE [46] and LHCb [57] in p-Pb collisions at $\sqrt{s_{\mathrm{NN}}}=5.02 \mathrm{TeV}$. At forward rapidity suppression of the inclusive $\Upsilon(1 \mathrm{~S})$ yield in $\mathrm{p}-\mathrm{Pb}$ collisions with respect to the yield from $\mathrm{p}-\mathrm{p}$ collisions scaled by the number of binary nucleon-nucleon collisions is observed. But at backward rapidity the suppression is less (Figure 7).

The results are compared with several theoretical model calculations $[54,55]$ including partonic energy loss effects with and without nuclear shadowing. Only models with energy loss plus shadowing could describe the data at forward rapidity but these models underestimate the suppression at backward rapidity.

The production of charmonia and bottomonia is the object of intense theoretical and experimental investigations. Their production mechanism in p-p collisions is described by models based on Quantum Chromodynamics (QCD) and gives reference for comparison with $\mathrm{p}-\mathrm{A}$ and $\mathrm{A}-\mathrm{A}$ data. At the LHC in $\mathrm{Pb}-\mathrm{Pb}$ collisions, the evidence for additional $J / \psi$ production from regeneration at low $p_{T}$ and strong $J / \psi$ suppression at large transverse momentum was obtained. For $\mathrm{p}-\mathrm{Pb}$ collisions theoretical models including nuclear suppression, parton shadowing, and coherent energy loss effects could reproduce $J / \psi$ production but fail to describe additional suppression of $\psi(2 S)$ and underestimate the observed $\Upsilon(1 S)$ suppression at forward rapidity.

At the LHC and RHIC the collective and hot nuclear matter effects may also be present in $\mathrm{p}-\mathrm{Pb}$ at $5.02 \mathrm{TeV}$ and in $\mathrm{d}$-Au collisions at $200 \mathrm{GeV}$ [35]. Therefore, the measurement of nuclear effects at lower energies and assuming the absence of the QGP formation in p-p and p-A collisions could give a reference for the study of hot nuclear matter effects. Moreover, the contribution of recombination process is small at low energies. The RHIC heavy flavor program with the energy scan can perform this investigation but, unfortunately, the luminosity at RHIC strongly decreases with decreasing the energy of collision. 


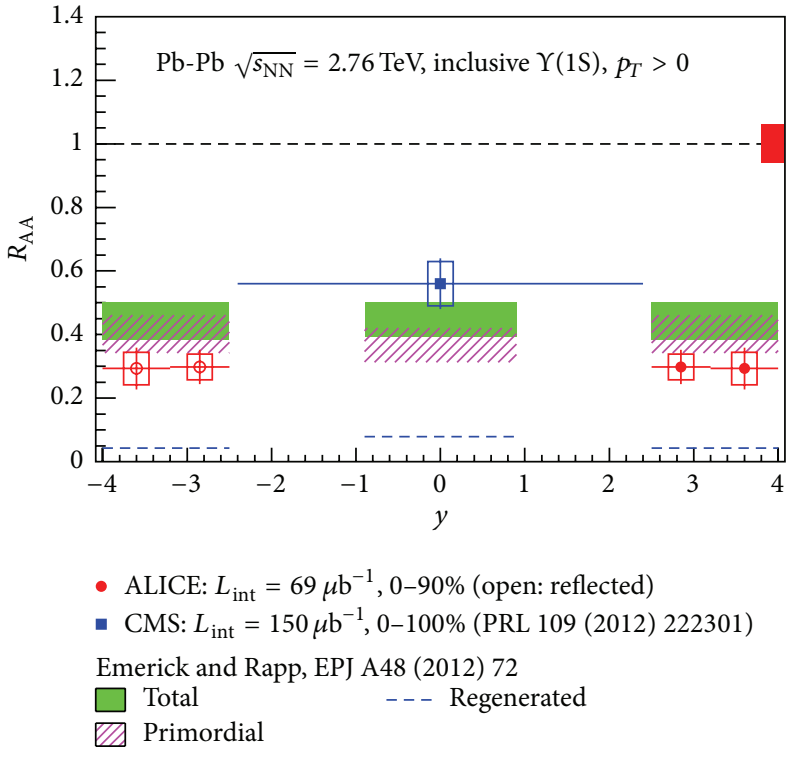

(a)

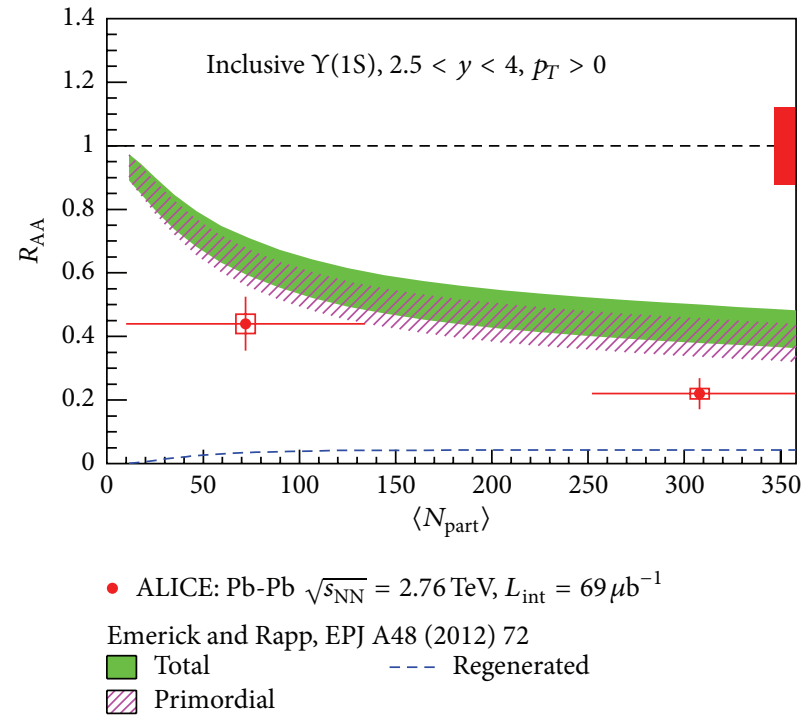

(b)

FIGURE 6: Comparison with theoretical predictions ALICE and CMS $R_{\mathrm{PbPb}}$ results for $\Upsilon(1 \mathrm{~S})$ versus rapidity (a) and ALICE results versus $N_{\text {part }}$ (b).

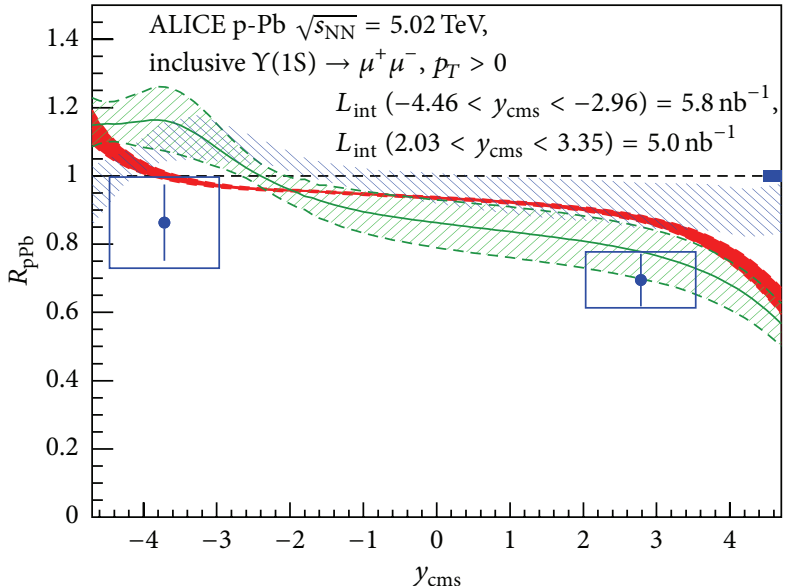

CEM + EPS09 NLO (Vogt, arXiv: 1301.3395 and priv.comm.)

Eloss (Arleo and Peigné, JHEP 1303 (2013) 122):

ELoss

ELoss + EPS09 NLO

Figure 7: Comparison of ALICE $R_{\mathrm{pPb}}$ results for $\Upsilon(1 \mathrm{~S})$ versus rapidity with theoretical predictions.

\section{Quarkonium Production at Fixed Target at the LHC Beams}

The energy intervals between the SPS, the RHIC, and the LHC are very important to study the mechanism of quarkonium production and suppression and for the investigation of the medium effects and conditions of the Quark-Gluon Plasma formation. Investigation of the cold nuclear matter effect and understanding the properties of matter require systematic and high statistics measurements of quarkonium production also in the low energy region. To clarify the CNM effects it will be possible to study the mechanism of quarkonium production and suppression with high statistics at low energies up to $35 \mathrm{GeV} /$ per nucleon in the CBM experiment at FAIR [63] and in the MPD experiment at NICA collider in Dubna [64]. At the CERN SPS, the fixed target experiment CHIC (Charm in Heavy Ion Collisions) for charmonium study at energy up to $\sqrt{s_{\mathrm{NN}}} \sim 20 \mathrm{GeV}$ is under preparation [65]. The Beam Energy Scan (BES-I) program at RHIC was performed by STAR and PHENIX collaborations. At STAR there is an ongoing fixed target program, with data already taken in the gold target test during $14.5 \mathrm{GeV}$ Au-Au run in 2014 with $\sqrt{s_{\mathrm{NN}}}=3.9 \mathrm{GeV}$ [66].

If the LHC proton and ion beams would be used with fixed targets, the energy below the nominal RHIC energy $(200 \mathrm{GeV})$ in $\mathrm{p}-\mathrm{A}$ and A-A collisions could be investigated. For $7 \mathrm{TeV}$ proton beam, the energy in N-N c.m. is $\sqrt{s_{\mathrm{NN}}}=$ 114.6 GeV, and for $\mathrm{Pb}$ beam at $2.75 \mathrm{TeV}$ it is $\sqrt{s_{\mathrm{NN}}}=71.8 \mathrm{GeV}$. By using the LHC beams with reduced energy, the data could be taken in the range $30-100 \mathrm{GeV}$. QCD phase diagram may have interesting features probed by $10-100 \mathrm{GeV}$ beams on fixed target. Search for signature of the phase transition and location of the critical point is the main goal of energy scan. Moreover, this is a possibility of investigating the mechanism of charmonium, $J / \psi$, and $\psi(2 S)$ productions. It will be possible to separate the mechanism of hard production and then suppression by hadronic dissociation in QGP from secondary production with recombination, since the probability of recombination could decrease with decreasing energy of collision like in thermal model [67].

The existing system to inject the gas target for the luminosity measurement (SMOG) at LHCb experiment could be used for fixed target physics [68]. There are some testing measurements of $\mathrm{p}-\mathrm{Ne}$ and $\mathrm{Pb}-\mathrm{Ne}$ collisions, but, for 
the moment, there are no precise measurements of the gas density and pressure.

The main parameter for quarkonium production measurement in the fixed target experiment is the acceptance. In order to study the feasibility of using the fixed target at the LHC for charmonium production, the geometrical acceptances for $J / \psi$ production on fixed target by means of AliRoot-FAST simulations were obtained. The detailed description of the calculation was given in [69]. For the model of quarkonium production the phenomenological Color Octet Model (COM) was used [70, 71]. For prompt $J / \psi$ production the rapidity and transverse momentum distributions are obtained, respectively, as a parameterization of the COM predictions and by extrapolating to LHC energies the $J / \psi$ transverse momentum $\left(p_{T}\right)$ distribution, measured at midrapidity by the CDF experiment at energy $\sqrt{s_{\mathrm{NN}}}=$ $1.8 \mathrm{TeV}$. The geometrical acceptances of the $J / \psi$ production for PHENIX at RHIC and fixed target experiments NA50 at SPS and HERA-B were also calculated for comparison [69].

5.1. Geometrical Acceptances for Fixed Target $\mathrm{Pb}-\mathrm{Pb}$ Collisions at the Energy $T=2.75 \mathrm{TeV}$ per Nucleon, $\sqrt{s_{\mathrm{NN}}}=71.8 \mathrm{GeV}$. The geometrical acceptance for the $J / \psi$ production at ALICE dimuon spectrometer was calculated by the beam axis at the interaction point $z=0$, at the points $z=+50 \mathrm{~cm}$ (in the direction to the dimuon spectrometer) and $z=-50 \mathrm{~cm}$ (outside the ITS) and at point $z=+350 \mathrm{~cm}$. The transverse momentum distribution for $J / \psi$ was generated using $p_{T}$ spectra in the CDF form, the same as for HERA and PHENIX, consistent with COM:

$$
\frac{d N}{d p_{T}} \sim p_{T}\left[1+\left(\frac{35 \pi p_{T}}{256\left\langle p_{T}\right\rangle}\right)^{2}\right]^{-6}
$$

For rapidity distribution the Gaussian spectra were used. The parameters of distribution were energy scaled. For $\mathrm{Pb}-\mathrm{Pb}$ collisions $p_{T}$ spectra with the value $\left\langle p_{T}\right\rangle=1.4$ and Gaussian rapidity distribution with mean values $y_{\mathrm{cm}}=0$ and $\sigma=1.1$ were used. The acceptances at $z=+50 \mathrm{~cm}$ and $z=-50 \mathrm{~cm}$ are approximately the same, but at point $z=+350 \mathrm{~cm}$ the geometrical acceptance is much less. The results were shown in [69].

In Figure 8, the $\mathrm{Pb}-\mathrm{Pb}$ results for $z=0$ and $z=+50 \mathrm{~cm}$ are plotted. The $J / \psi$ are accepted in the rapidity range $-2.5<\eta<$ $-4.0(-2.97<\eta<-4.09)$ for $J / \psi$ production at the point $z=$ $0(z=+50 \mathrm{~cm})$. The geometrical acceptances, $I$, are equal to $(12.0 \pm 0.2) \%$ for $J / \psi$ production at $z=0$ point and $(8.0 \pm 0.2)$ $\%$ at $z=+50 \mathrm{~cm}$.

5.2. Geometrical Acceptances for Fixed Target $p-p$ and $p-A$ Collisions at the Energy $T=7 \mathrm{TeV}$, Corresponding to $\sqrt{s_{\mathrm{NN}}}=$ $114.6 \mathrm{GeV}$. The $J / \psi$ productions in $\mathrm{p}-\mathrm{p}$ and $\mathrm{p}-\mathrm{Pb}$ collisions are generated using $p_{T}$ spectra in the same form as for $\mathrm{Pb}-\mathrm{Pb}$ collision, but with the energy scaled parameter:

$$
\frac{d N}{d p_{T}} \sim p_{T}\left[1+\left(\frac{35 \pi p_{T}}{256\left\langle p_{T}\right\rangle}\right)^{2}\right]^{-6}
$$

where $\left\langle p_{T}\right\rangle=1$.6. For rapidity distribution the Gaussian spectra were used with mean value being equal to $y_{\mathrm{cm}}=0$ and $\sigma=1.25$. Geometrical acceptances for $J / \psi$ production were calculated at points $z=0, z=+50 \mathrm{~cm}$, and $z=$ $-50 \mathrm{~cm}$ (outside the ITS) and at point $z=+350 \mathrm{~cm}$. The results were shown in [69]. The acceptances at $z=+50 \mathrm{~cm}$ and $z=$ $-50 \mathrm{~cm}$ are roughly the same, but at point $z=+350 \mathrm{~cm}$ the geometrical acceptance is much less. In Figure 9, the results for $z=0$ and $z=+50 \mathrm{~cm}$ are plotted.

The geometrical acceptances, $I$, are equal to $(8.5 \pm 0.2) \%$ for $J / \psi$ production at $z=0$ point and $(6.0 \pm 0.3) \%$ at $z=$ $+50 \mathrm{~cm}$. We have calculated also the geometrical acceptances for $J / \psi$ with the cut on transverse momentum of the single muon $p_{T}>1 \mathrm{GeV} / \mathrm{c}$. The calculated geometrical acceptances for fixed target measurements are of the same order and even larger than geometrical acceptances for colliding nuclei in ALICE [69].

5.3. Luminosity, Cross Sections, and Counting Rates for $p-p$, $p$ $\mathrm{A}$, and $\mathrm{Pb}-\mathrm{Pb}$ Collisions. The target in the form of thin ribbon could be placed around the main orbit of the LHC, as it was already used for the experiment on collider with a fixed target at HERA-B [72]. The life time of the beam is determined by the beam-beam and beam-gas interactions. Therefore, after some time the particles will leave the main orbit and interact with the target ribbon. So for fixed target measurements only halo of the beam will be used. Therefore, no deterioration of the main beam will be introduced. The experiments at other interaction points will not feel any presence of the fixed target. Since the target ribbon should not interfere during the beam formation and acceleration process it should be lifted in the working position after the tuning of the beam.

In the ALICE measurements in 2011 in $p$-p run it was $1.2 \cdot 10^{11}$ protons per bunch, 1380 bunches and life time of 14.5 hours. From these parameters one can estimate the particle loss of $1.1 \cdot 10^{13}$ protons during one hour $\left(3.1 \cdot 10^{9}\right.$ protons $\left./ \mathrm{s}\right)$ and mean luminosity about $3 \cdot 10^{30} \mathrm{~cm}^{-2} \mathrm{~s}^{-1}$ for 500 -micron lead ribbon. For $\mathrm{Pb}$ beam it was $1 \cdot 10^{8}$ ions per bunch, 358 bunches and life time of 6.5 hours. The particle loss is about $5.1 \cdot 10^{9}$ ions during one hour $\left(1.4 \cdot 10^{6}\right.$ ions/s). The mean luminosity is about $1.7 \cdot 10^{27} \mathrm{~cm}^{-2} \mathrm{~s}^{-1}$ for 500 -micron lead ribbon. The luminosity estimates are shown in Table 1 . The value of the nucleon-nucleon charmonium total production cross section shown in Table 1 for $14 \mathrm{TeV}$ was calculated by CEM (Color Evaporation Model) [73] with MRST HO PDF. The cross sections for lower energies were obtained by interpolation of proton-proton cross sections measured at RHIC at $\sqrt{\mathrm{s}_{\mathrm{NN}}}=$ $200 \mathrm{GeV}$ and at SPS in the NA51 experiment at $450 \mathrm{GeV}$ per nucleon $\left(\sqrt{s_{\mathrm{NN}}}=29.1 \mathrm{GeV}\right)$, and were extracted from the data of the NA50 experiment at $\sqrt{\mathrm{s}_{\mathrm{NN}}}=27.4 \mathrm{GeV}$ for proton-lead collisions.

The high statistics results could be obtained by fixed target experiments on $J / \psi$ production in $\mathrm{p}-\mathrm{Pb}$ and $\mathrm{Pb}-\mathrm{Pb}$ collisions with the counting rate values presented in Table 1 . For $\mathrm{Pb}$ $\mathrm{Pb}$ collisions, luminosity is smaller but the production cross section is larger. The measurement of $\psi(2 \mathrm{~S})$ production is also feasible with better statistical accuracy than at RHIC collider.

The fixed target experiment AFTER using the LHC beams extracted by a bent crystal was proposed [74]. The experiment 

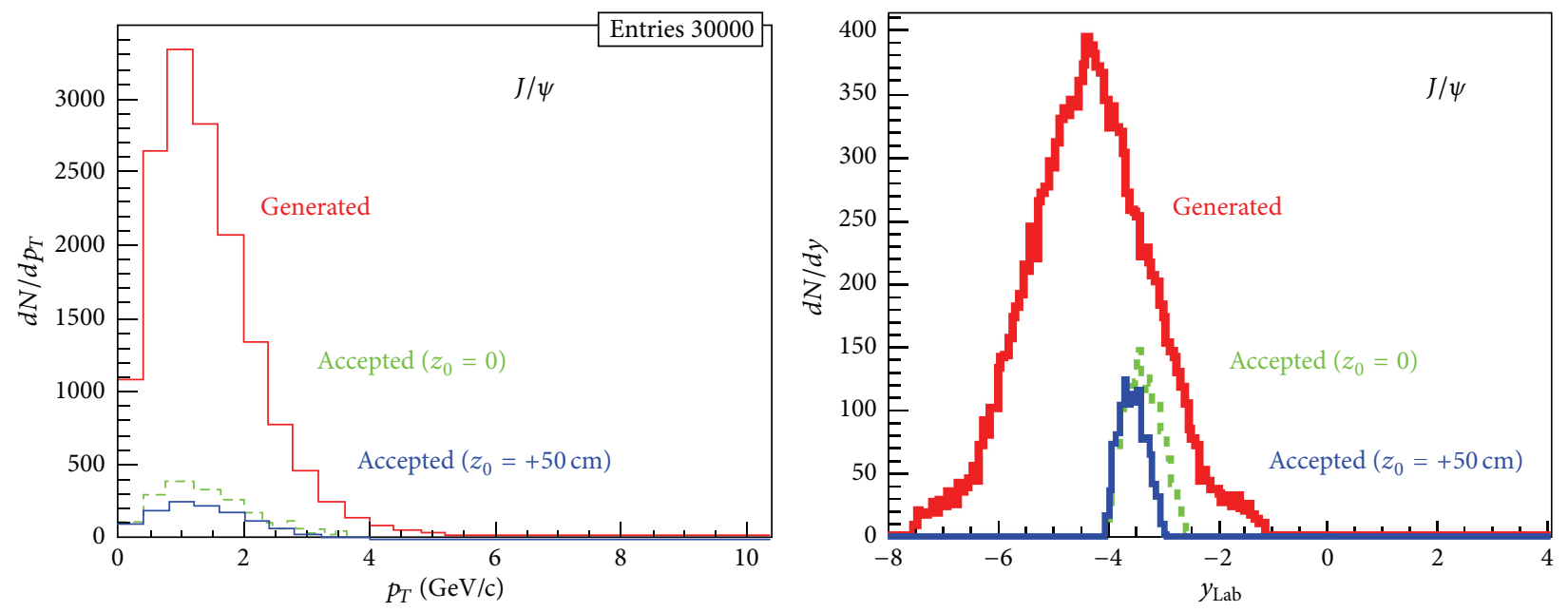

(a)
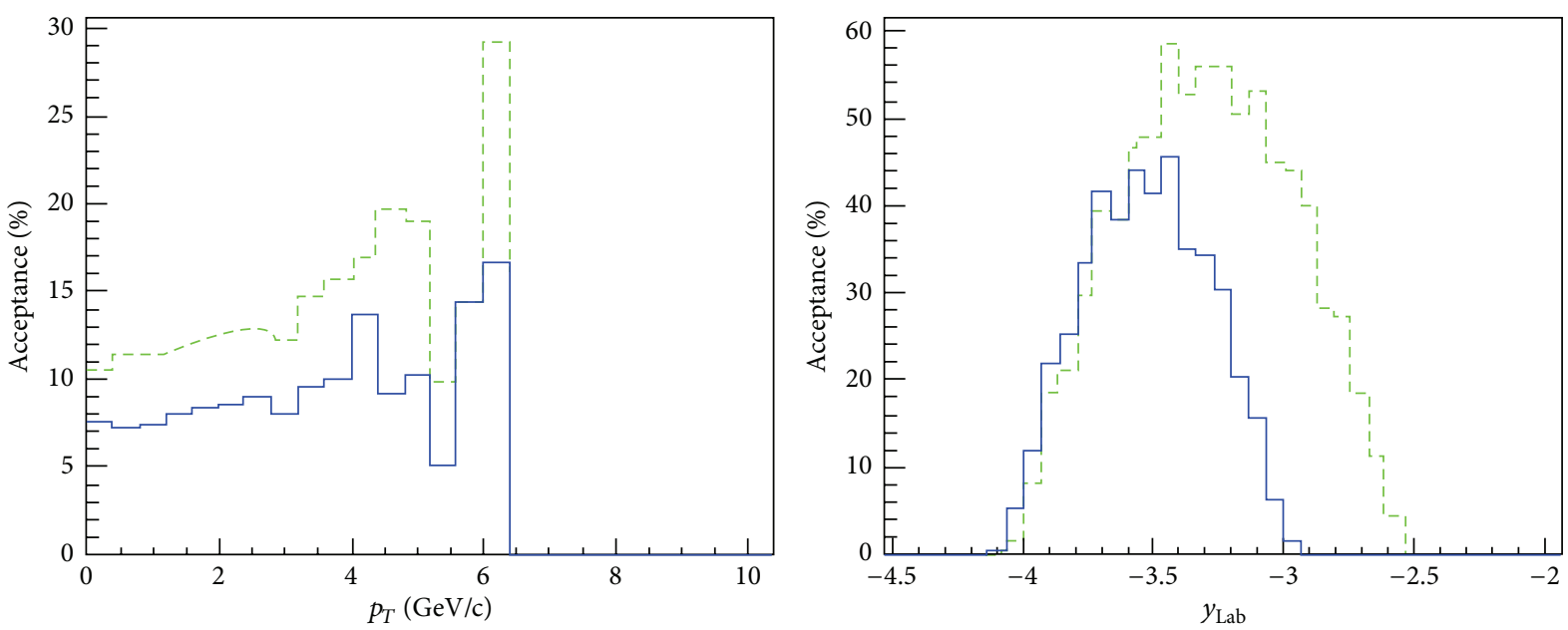

(b)

FiguRE 8: Transverse momentum and rapidity distributions for $J / \psi$ events, produced at $z=+50 \mathrm{~cm}$ (solid lines) and $z=0$ (dashed lines) in fixed target $\mathrm{Pb}-\mathrm{Pb}$ collisions at $\sqrt{s}=71.8 \mathrm{GeV}$ per nucleon pair (a) and corresponding acceptances (b).

TABLE 1: Luminosity, cross sections $\left(x_{F}>0\right)$, and counting rates.

\begin{tabular}{|c|c|c|c|c|c|c|c|}
\hline System & $\sqrt{s}, \mathrm{TeV}$ & $\sigma_{\mathrm{NN}}, \mu \mathrm{b}$ & $\begin{array}{c}\sigma_{\mathrm{pA}}, \mu \mathrm{b} \\
\left(\mathrm{A}^{0.92} \sigma_{\mathrm{NN}}\right)\end{array}$ & $I, \%$ & $\mathrm{IB} \sigma_{\mathrm{pA}}, \mu \mathrm{b}$ & $L, \mathrm{~cm}^{-2} \mathrm{~s}^{-1}$ & Rate, $\mathrm{h}^{-1}$ \\
\hline $\mathrm{pp}$ & 14 & 54.1 & 54.1 & 4.7 & 0.150 & $3 \cdot 10^{30}$ & 1620 \\
\hline $\mathrm{pp}_{\mathrm{RHIC}}$ & 0.200 & 2.7 & 2.7 & 3.6 & 0.0057 & $1 \cdot 10^{31}$ & 205 \\
\hline $\mathrm{pPb}_{\mathrm{NA50}}$ & 0.0274 & 0.19 & 25.7 & 14.0 & 0.212 & $7 \cdot 10^{29}$ & 535 \\
\hline $\mathrm{pPb}_{\text {fixed }}$ & 0.1146 & 0.65 & 80.2 & 6.0 & 0.310 & $3 \cdot 10^{30^{*}}$ & 3360 \\
\hline $\mathrm{pPb}_{\text {fixed }}$ & 0.0718 & 0.55 & 74.6 & 8.0 & 0.349 & $3 \cdot 10^{30 *}$ & 3780 \\
\hline $\mathrm{PbPb}_{\text {fixed }}$ & 0.0718 & 0.55 & 11970 & 8.0 & 42.9 & $1.7 \cdot 10^{27^{* *}}$ & 292 \\
\hline
\end{tabular}

${ }^{*} \mathrm{pPb}_{\text {fixed }}, 500 \mu$ wire, $3.1 \cdot 10^{9}$ protons/s.

${ }^{* *} \mathrm{PbPb}_{\text {fixed }}, 500 \mu$ wire, $1.4 \cdot 10^{6}$ ions/s.

AFTER has a wide physical program and gives possibility of using different targets with high thickness, so it gets higher luminosity (20 times more for $1 \mathrm{~cm}$ target versus $500 \mu \mathrm{m}$ ). But the experiment AFTER demands a lot of space for installation and has high cost.
Fixed target experiment with the target in the form of thin ribbon may be placed at the existing experimental installation (e.g., LHCb). The target could be lifted in the working position with the aid of rotation system only after beam tuning. This experiment will use only halo of the beam 

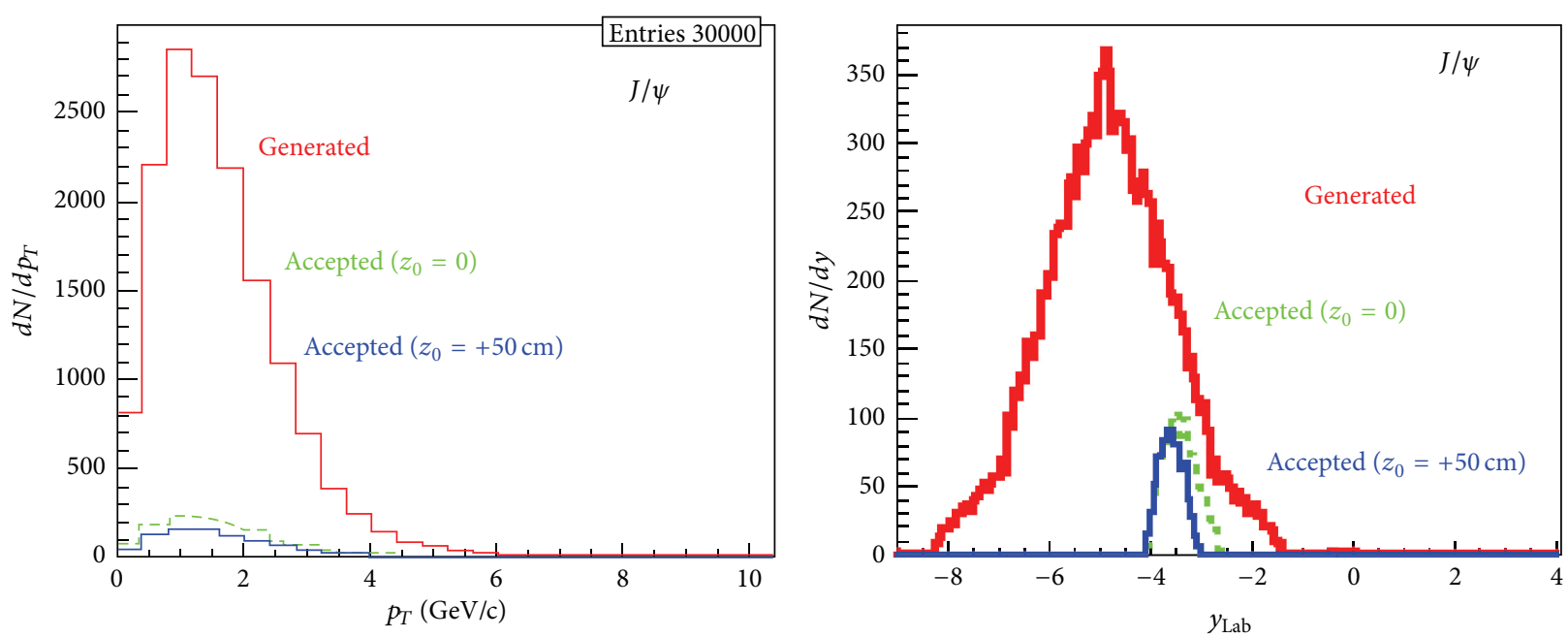

(a)
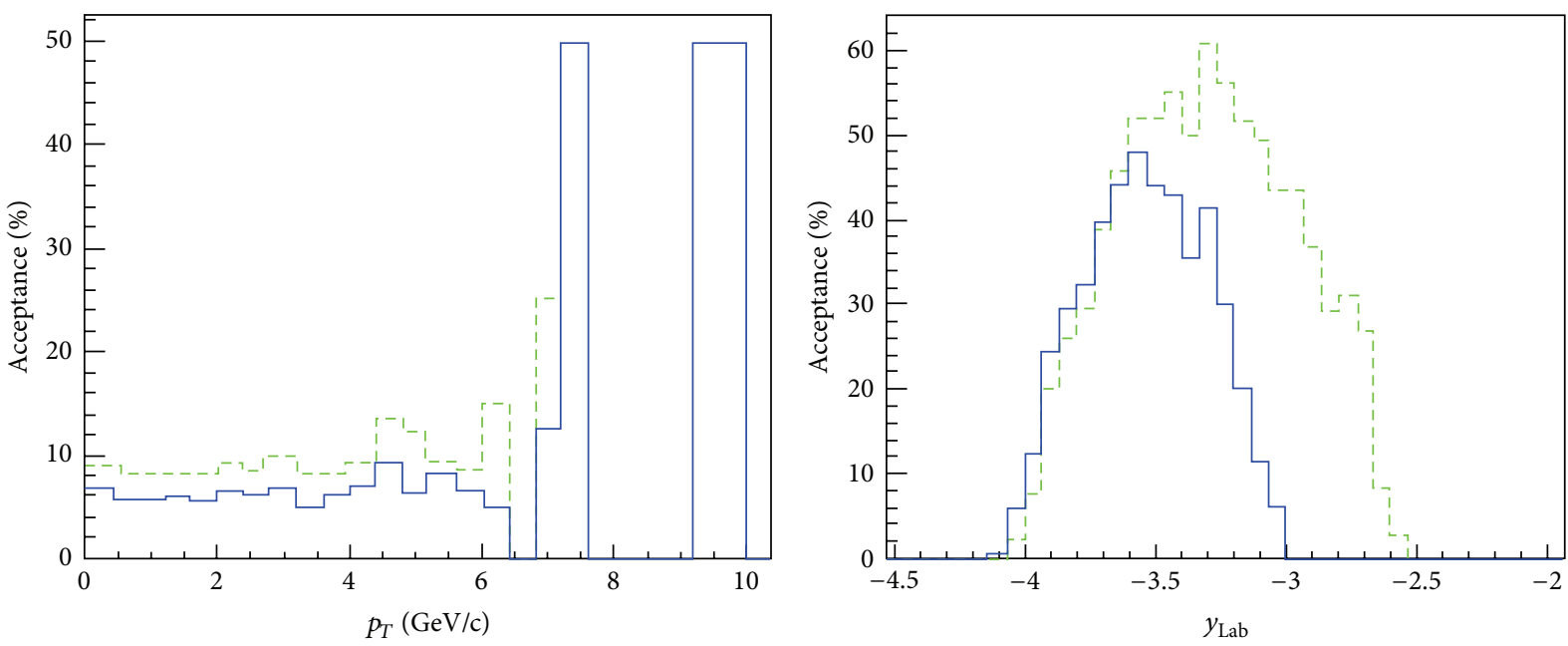

(b)

FIGURE 9: Transverse momentum and rapidity distributions for $J / \psi$ events, produced at $z=+50 \mathrm{~cm}$ (solid line) and at $z=0$ (dashed line) in fixed target p-A collisions at $\sqrt{s_{\mathrm{NN}}}=114.6 \mathrm{GeV}$ per nucleon pair (a) and corresponding acceptances (b).

(the target could be used as extra collimator). This fixed target experiment with the target in the form of thin ribbon looks like a first stage of more complicated experiment AFTER.

\section{Conclusion}

The fixed target experiment at LHC will give possibility for precise quarkonium studies at energies below nominal RHIC energy $(200 \mathrm{GeV})$. It has advantage of high luminosity compared to collider experiments. The use of fixed target at LHC could provide in a short time the data for different targets and maybe for different projectile nuclei with high statistics. By using the LHC beams the data could be taken in the range $\sqrt{s_{\mathrm{NN}}}=29-115 \mathrm{GeV}$. Search for signature of the phase transition and location of the critical point is the main goal of energy scan. Moreover, this is a possibility of investigating the mechanism of charmonium, $J / \psi$, and $\psi(2 S)$ productions to separate the possibilities of hard production and then suppression by hadronic dissociation in QGP or secondary production with recombination. Additional $J / \psi$ mesons are expected to be produced from deconfined charm quarks by kinetic recombination in the QGP or by statistical hadronization at the phase boundary. The probability of recombination decreases with decreasing energy of collision. Therefore, the important information about mechanism of charmonium production and possible QGP formation could be obtained. The experiment with the fixed target in the form of thin ribbon looks like a first stage of more complicated experiment AFTER on the LHC beams.

\section{Conflict of Interests}

The authors declare that there is no conflict of interests regarding the publication of this paper. 


\section{References}

[1] T. Matsui and H. Satz, "J/ $\psi$ suppression by quark-gluon plasma formation," Physics Letters B, vol. 178, no. 4, pp. 416-422, 1986.

[2] M. C. Abreu, J. Astruc, C. Baglin et al., "J/ $\psi$ and $\psi^{\prime}$ production in $\mathrm{p}, \mathrm{O}$ and $\mathrm{S}$ induced reactions at SPS energies," Physics Letters B, vol. 466, no. 2-4, pp. 408-414, 1999.

[3] M. C. Abreu, B. Alessandro, C. Alexa et al., "Anomalous J/ $\psi$ suppression in $\mathrm{Pb}-\mathrm{Pb}$ interactions at $158 \mathrm{GeV} / \mathrm{c}$ per nucleon," Physics Letters B, vol. 410, no. 2-4, pp. 337-343, 1997.

[4] B. Alessandro, C. Alexa, R. Arnaldi et al., "A new measurement of $J / \psi$ suppression in $\mathrm{Pb}-\mathrm{Pb}$ collisions at $158 \mathrm{GeV}$ per nucleon," The European Physical Journal C, vol. 39, pp. 335-345, 2005.

[5] B. Alessandro, C. Alexa, R. Arnaldi et al., "Charmonia and Drell-Yan production in proton-nucleus collisions at the CERN SPS," Physics Letters B, vol. 553, pp. 167-178, 2003.

[6] B. Alessandro, C. Alexa, R. Arnaldi et al., " $J / \psi$ and $\psi$ ' production and their normal nuclear absorption in proton-nucleus collisions at $400 \mathrm{GeV}$,' The European Physical Journal C, vol. 48, no. 2, pp. 329-341, 2006.

[7] B. Alessandro, C. Alexa, R. Arnaldi et al., " $\psi$ ' production in $\mathrm{Pb}-\mathrm{Pb}$ collisions at $158 \mathrm{GeV} /$ nucleon," The European Physical Journal C, vol. 49, no. 2, pp. 559-567, 2007.

[8] R. Arnaldi and The NA60 Collaboration, "J/ $\psi$ production in p-A and A-A collisions at fixed target experiments," Nuclear Physics A, vol. 830, no. 1-4, pp. 345c-352c, 2009.

[9] B. Alessandro, C. Alexa, R. Arnaldi et al., " $J / \psi$ production in proton-nucleus collisions at 158 and $400 \mathrm{GeV}$," Physics Letters B, vol. 706, no. 4-5, pp. 263-267, 2012.

[10] I. Abt, M. Adams, M. Agari et al., "Kinematic distributions and nuclear effects of $J / \psi$ production in $920 \mathrm{GeV}$ fixed-target proton-nucleus collisions," The European Physical Journal C, vol. 60, no. 4, pp. 525-542, 2009.

[11] M. J. Leitch, W. M. Lee, M. E. Beddo et al., "Measurement of differences between $J / \psi$ and $\psi /$ suppression in $p-A$ collisions," Physical Review Letters, vol. 84, no. 15, pp. 3256-3260, 2000.

[12] A. Adare, S. Afanasiev, C. Aidala et al., " $J / \psi$ Production versus centrality, transverse momentum, and rapidity in $\mathrm{Au}+\mathrm{Au}$ collisions at $\sqrt{s_{N N}}=200 \mathrm{GeV}$," Physical Review Letters, vol. 98, Article ID 232301, 2007.

[13] A. Adare, S. Afanasiev, C. Aidala et al., "J/ $\psi$ suppression at forward rapidity in $\mathrm{Au}+\mathrm{Au}$ collisions at $\sqrt{s_{N N}}=200 \mathrm{GeV}$," Physical Review C, vol. 84, Article ID 054912, 2011.

[14] A. Adare, S. Afanasiev, C. Aidala et al., "Ground and excited state charmonium production in $p+p$ collisions at $\sqrt{s}=$ 200 GeV," Physical Review D, vol. 85, no. 9, Article ID 092004, 27 pages, 2012.

[15] A. Adare, S. Afanasiev, C. Aidala et al., " $J / \psi$ production in $\sqrt{s_{N N}}=200 \mathrm{GeV} \mathrm{Cu}+\mathrm{Cu}$ collisions," Physical Review Letters, vol. 101, Article ID 122301, 2008.

[16] A. Adare, S. Afanasiev, C. Aidala et al., "Cold nuclear matter effects on $\mathrm{J} / \psi$ yields as a function of rapidity and nuclear geometry in $\mathrm{d}+\mathrm{A}$ collisions at $\sqrt{s_{N N}}=200 \mathrm{GeV}$," Physical Review Letters, vol. 107, Article ID 142301, 2011.

[17] L. Adamczyk, J. K. Adkins, G. Agakishiev et al., " $J / \psi$ production at low $p T$ in $\mathrm{Au}+\mathrm{Au}$ and $\mathrm{Cu}+\mathrm{Cu}$ collisions at $\sqrt{s_{N N}}=200 \mathrm{GeV}$ with the STAR detector," Physical Review C, vol. 90, no. 2, Article ID 24906, 13 pages, 2014.

[18] J. Adams, C. Adler, M. M. Aggarwal et al., "J/ $\psi$ production at high transverse momenta in $\mathrm{p}+\mathrm{p}$ and $\mathrm{Au}+\mathrm{Au}$ collisions at $\sqrt{s_{N N}}=200 \mathrm{GeV}$," Physics Letters B, vol. 722, pp. 55-62, 2013.
[19] B. I. Abelev, M. Aggarwal, Z. Ahammed, and etaL, " $J / \psi$ production at high transverse momenta in $p+p$ and $\mathrm{Cu}+$ Cu collisions at $\sqrt{s_{N N}}=200 \mathrm{GeV}$," Physical Review C, vol. 80, Article ID 041902(R), 2009.

[20] H. Brambilla, S. Eidelman, B. K. Heltsley et al., "Heavy quarkonium: progress, puzzles, and opportunities," The European Physical Journal C, vol. 71, article 1534, 2011.

[21] R. L. Thews, "Quarkonium formation in statistical and kinetic models," The European Physical Journal C, vol. 43, no. 1-4, pp. 97-102, 2005.

[22] R. L. Thews and M. L. Mangano, "Momentum spectra of charmonium produced in a quark-gluon plasma," Physical Review C, vol. 73, no. 1, Article ID 014904, 2006.

[23] A. Andronic, P. Braun-Munzinger, K. Redlich, and J. Stachel, "Charmonium and open charm production in nuclear collisions at SPS/FAIR energies and the possible influence of a hot hadronic medium," Physics Letters B, vol. 659, no. 1-2, pp. 149$155,2008$.

[24] P. Braun-Munzinger and J. Stachel, "(Non)thermal aspects of charmonium production and a new look at $J / \psi$ suppression," Physics Letters B, vol. 490, no. 3-4, pp. 196-202, 2000.

[25] K. Aamodt, A. Abrahantes Quintana, D. Adamová et al., "Erratum to 'Rapidity and transverse momentum dependence of inclusive $J / \psi$ production in pp collisions at $\sqrt{s}=7 \mathrm{TeV}$, Physics Letters B, vol. 704, no. 5, p. 442, 2011," Physics Letters B, vol. 718, no. 2, pp. 692-698, 2012.

[26] B. Abelev, J. Adam, D. Adamova et al., "Centrality, rapidity and transverse momentum dependence of $\mathrm{J} / \psi$ suppression in $\mathrm{Pb}-\mathrm{Pb}$ collisions at $\sqrt{s_{N N}}=2.76$," Physics Letters B, vol. 734, pp. 314327, 2014.

[27] S. Chatrchyan, V. Khachatryan, A. M. Sirunyan et al., " $J / \psi$ and $\psi(2 S)$ production in pp collisions at $\sqrt{s}=7$ Tev," Journal of High Energy Physics, vol. 2012, article 11, 2012.

[28] S. Chatrchyan, V. Khachatryan, A. M. Sirunyan et al., "Suppression of non-prompt $\mathrm{J} / \psi$, prompt $\mathrm{J} / \psi$, and $\Upsilon(1 \mathrm{~S})$ in $\mathrm{PbPb}$ collisions at $\sqrt{s_{N N}}=2.76 \mathrm{TeV}$," Journal of High Energy Physics, vol. 2012, no. 5, article 63, 2012.

[29] G. Aad, B. Abbott, J. Abdallah et al., "Measurement of the differential cross-sections of inclusive, prompt and non-prompt $J / \psi$ production in proton-proton collisions at $\sqrt{s}=7 \mathrm{TeV}$,' Nuclear Physics B, vol. 850, no. 3, pp. 387-444, 2011.

[30] G. Aad, B. Abbott, J. Abdallah et al., "Measurement of the centrality dependence of $J / \psi$ yields and observation of $\mathrm{Z}$ production in lead-lead collisions with the ATLAS detector at the LHC," Physics Letters B, vol. 697, no. 4, pp. 294-312, 2011.

[31] R. Aaij, B. Adeva, M. Adinolfi et al., "Measurement of $J / \psi$ production in $p p$ collisions at $\sqrt{s}=7 \mathrm{TeV}$," The European Physical Journal C, vol. 71, article 1645, 2011.

[32] R. Aaij, C. A. Beteta, A. Adametz et al., "Measurement of $J / \psi$ production in $p p$ collisions at $\sqrt{s}=2.76 \mathrm{TeV}$," Journal of High Energy Physics, vol. 2013, no. 02, article 041, 2013.

[33] B. Abelev, J. Adam, D. Adamova et al., "Measurement of prompt $J / \psi$ and beauty hadron production cross sections at midrapidity in pp collisions at $\sqrt{s}=7 \mathrm{TeV}$," Journal of High Energy Physics, vol. 2012, no. 11, article 065, 2012.

[34] J. Badier, J. Boucrot, J. Bourotte et al., "Experimental $J / \psi$ hadronic production from 150 to $280 \mathrm{GeV} / \mathrm{c}$," Zeitschrift für Physik C, vol. 20, no. 2, pp. 101-106, 1983.

[35] A. D. Frawley, "PHENIX charmonia: what have we learned from d+Au collisions?” Nuclear Physics A, vol. 932, pp. 105-110, 2014. 
[36] X. Zhao and R. Rapp, "Charmonium in medium: from correlators to experiment," Physical Review C, vol. 82, Article ID 064905, 2010.

[37] Y. Liu, Z. Qu, N. Xu, and P. Zhuang, "J/ $\psi$ transverse momentum distribution in high energy nuclear collisions," Physics Letters $B$, vol. 678, no. 1, pp. 72-76, 2009.

[38] A. Adare, C. Aidala, N. N. Ajitanand et al., "Nuclear matter effects on $\mathrm{J} / \psi$ production in asymmetric $\mathrm{Cu}+\mathrm{Au}$ collisions at $\sqrt{s_{N N}}=200$ GeV," Physical Review C, vol. 90, Article ID 064908, 2014.

[39] A. Iordnova, "Nuclear matter effects on $\mathrm{J} / \psi$ production in $\mathrm{Cu}+\mathrm{Au}$ and $U+U$ collisions in PHENIX," in Proceedings of the International Workshop on Deep-Inelastic Scattering and Related Subjects (DIS '14), p. 191, 2014.

[40] W. Zha, "Recent measurements of quarkonium production in $\mathrm{p}$ $+\mathrm{p}$ and A + A collisions from the STAR experiment," Nuclear Physics A, vol. 931, pp. 596-600, 2014.

[41] A. Adare, C. Aidala, N. N. Ajitanand et al., " $J / \psi$ suppression at forward rapidity in $\mathrm{Au}+\mathrm{Au}$ collisions at $\sqrt{s_{N N}}=39$ and 62.4 GeV," Physical Review C, vol. 86, no. 6, Article ID 064901, 10 pages, 2012.

[42] B. Abelev, J. Adam, D. Adamová et al., "Measurement of quarkonium production at forward rapidity in $\mathrm{pp}$ collisions at $\sqrt{s}=7$ TeV," The European Physical Journal C, vol. 74, article 2974, 2014.

[43] B. Abelev, J. Adam, D. Adamová et al., "J/ $\psi$ suppression at forward rapidity in $\mathrm{Pb}-\mathrm{Pb}$ collisions at $\sqrt{s_{N N}}=2.76 \mathrm{TeV}$," Physical Review Letters, vol. 109, Article ID 072301, 2012.

[44] B. Abelev, J. Adam, D. Adamová et al., "J/ $\psi$ production and nuclear effects in $\mathrm{p}-\mathrm{Pb}$ collisions at $\sqrt{s_{\mathrm{NN}}}=5.2 \mathrm{TeV}$," Journal of High Energy Physics, vol. 2014, no. 2, article 73, 2014.

[45] B. Abelev, J. Adam, D. Adamová et al., " $J / \psi$ Production and nuclear effects in p-Pb collisions at $\sqrt{s_{\mathrm{NN}}}=5.02 \mathrm{TeV}$," Journal of High Energy Physics, vol. 2014, no. 12, article 73, 2014.

[46] B. Abelev and ALICE Collaboration, "Production of inclusive $\Upsilon(1 \mathrm{~S})$ and $\Upsilon(2 \mathrm{~S})$ in $\mathrm{p}-\mathrm{Pb}$ collisions at $\sqrt{\mathrm{S}_{\mathrm{NN}}}=5.02 \mathrm{TeV}$," Physics Letters B, vol. 740, pp. 105-117, 2015.

[47] CMS Collaboration, "Measurement of the $\Upsilon(1 S), \Upsilon(2 S)$, and $\Upsilon(3 \mathrm{~S})$ cross sections in pp collisions at $\sqrt{s}=7 \mathrm{TeV}$," Physics Letters B, vol. 727, no. 1-3, pp. 101-125, 2013.

[48] S. Chatrchyan, V. Khachatryan, A. M. Sirunyan et al., "Observation of sequential $\Upsilon$ suppression in $\mathrm{PbPb}$ collisions," Physical Review Letters, vol. 109, no. 22, Article ID 222301, 15 pages, 2012.

[49] S. Chatrchyan, V. Khachatryan, A. M. Sirunyan et al., "Indications of suppression of excited $\Upsilon$ states in $\mathrm{Pb}-\mathrm{Pb}$ collisions at $\sqrt{s_{\mathrm{NN}}}=2.76 \mathrm{TeV}$, , Physical Review Letters, vol. 107, Article ID 052302, 2011.

[50] R. Aaij, B. Adeva, M. Adinolfi et al., "Study of $J / \psi$ production and cold nuclear matter effects in $p \mathrm{~Pb}$ collisions at $\sqrt{s_{N N}}=$ $5 \mathrm{TeV}$," Journal of High Energy Physics, vol. 2014, no. 2, article 72, 2014.

[51] R. Aaij, B. Adeva, M. Adinolfi et al., "Study of $\Upsilon$ production and cold nuclear matter effects in $p \mathrm{~Pb}$ collisions at $\sqrt{s_{N N}}=5 \mathrm{TeV}$," Journal of High Energy Physics, vol. 2014, no. 7, article 094, 2014.

[52] B. Abelev, J. Adam, D. Adamova et al., " $J / \psi$ production as a function of charged particle multiplicity in pp collisions at $\sqrt{s}=$ 7 Tev," Physics Letters B, vol. 712, no. 3, pp. 165-175, 2012.

[53] H. Fujii and K. Watanable, "Heavy quark pair production in high-energy pA collisions: quarkonium," Nuclear Physics A, vol. 915, pp. 1-23, 2013.
[54] J. L. Abbacete, N. Armesto, R. Baier et al., "Predictions for $p+P b$ collisions at $\sqrt{s_{N N}}=5 \mathrm{TeV}$,' International Journal of Modern Physics E, vol. 22, Article ID 1330007, 2013.

[55] F. Arleo and S. Peigné, "Heavy-quarkonium suppression in p-A collisions from parton energy loss in cold QCD matter," Journal of High Energy Physics, vol. 2013, no. 3, article 122, 2013.

[56] F. Arlelo, R. Kolevativ, S. Peigne, and M. Rustamova, "Centrality and $p_{\perp}$ dependence of $\mathrm{J} / \psi$ suppression in proton-nucleus collisions from parton energy loss," Journal of High Energy Physics, vol. 2013, no. 5, article 155, 2013.

[57] R. Aaij, B. Adeva, M. Adinolfi et al., "Study of $\Upsilon$ production and cold nuclear matter effects in $p \mathrm{~Pb}$ collisions at $\sqrt{s_{N N}}=5 \mathrm{TeV}$," Journal of High Energy Physics, vol. 2014, no. 7, article 94, 2014.

[58] R. Aaij, C. A. Beteta, B. Adeva et al., "Measurement of $\Upsilon$ production in $p p$ collisions at $\sqrt{s}=7 \mathrm{TeV}$," The European Physical Journal C, vol. 72, article 2025, 2012.

[59] V. Khachatryan, A. M. Sirunyan, A. Tumasyan et al., "Upsilon production cross section in $p p$ collisions at $\sqrt{s}=7 \mathrm{TeV}$," Physical Review D, vol. 83, no. 11, Article ID 112004, 27 pages, 2011.

[60] A. Emerick, X. Zhao, and R. Rapp, "Bottomonia in the quarkgluon plasma and their production at RHIC and LHC," The European Physical Journal A, vol. 48, article 72, 2012.

[61] B. Abelev, J. Adam, D. Adamova et al., "Suppression of $\Upsilon(1 S)$ at forward rapidity in $\mathrm{Pb}-\mathrm{Pb}$ collisions at $\sqrt{s_{\mathrm{NN}}}=2.76 \mathrm{Tev}$," Physics Letters B, vol. 738, pp. 361-372, 2014.

[62] A. Zhou, N. Xu, Z. Xu, and P. Zhuang, "Medium effects on charmonium production at ultrarelativistic energies available at the CERN Large Hadron Collider," Physical Review C, vol. 89, Article ID 054911, 2014.

[63] C. Hohne, "Measurement of dileptons with the CBM experiment at FAIR," Nuclear Physics A, vol. 931, pp. 735-739, 2014.

[64] A. Sissakian, A. Sorin, I. Meshkov et al., "Design and construction of Nuclotron-based Ion Collider fAcility (NICA)," Conceptual Design Report, JINR, Dubna, Russia, 2008.

[65] F. Fleuret, F. Arleo, E. G. Ferreiro, P.-B. Gossiauxd, and S. Peigné, "Expression of Interest for an experiment to study charm production with proton and heavy ion beams (CHIC: Charm in Heavy Ion Collisions)," CERN-SPSC-2012-031/IPCEOI-008, 2012.

[66] B. Haag, O. Hajkova, A. Hamed et al., "A fixed-target program for STAR: extending the low energy reach of the RHIC beam energy scan,” QM2014, Quark Matter Poster, 2014.

[67] R. Hagedorn, "Multiplicities, $p_{T}$ distributions and the expected hadron-quark-gluon phase transition," La Rivista del Nuovo Cimento, vol. 6, pp. 1-50, 1983.

[68] M. Ferro-Luzzi, "Luminosity and luminous region shape for pure Gaussian bunches," Tech. Rep. CERN-LHCb-PUB-2012016, 2012.

[69] A. B. Kurepin, N. S. Topilskaya, and M. B. Golubeva, "Charmonium production in fixed-target experiments with SPS and LHC beams at CERN," Physics of Atomic Nuclei, vol. 74, no. 3, pp. 446452, 2011.

[70] M. Kramer, "Quarkonium production at high-energy colliders," Progress in Particle and Nuclear Physics, vol. 47, no. 1, pp. 141-201, 2001.

[71] E. Braaten, S. Fleming, and A. K. Leibovich, "Nonrelativistic QCD analysis of bottomonium production at the Fermilab Tevatron," Physical Review D, vol. 63, no. 9, Article ID 094006, 2001. 
[72] K. Ehret, "Commissioning of the HERA-B internal target: using the HERA proton ring as a B-factory," Nuclear Instruments and Methods in Physics Research A, vol. 446, no. 1-2, pp. 190-198, 2000.

[73] V. D. Barger, W. Y. Keung, and R. J. Phillips, "On $\psi$ and $\Upsilon$ production via gluons," Physics Letters B, vol. 91, no. 2, pp. 253258, 1980.

[74] S. J. Brodsky, F. Fleuret, C. Hadjidakis, and J. P. Lansberg, "Physics opportunities of a fixed-target experiment using LHC beams," Physics Reports, vol. 522, no. 4, pp. 239-255, 2013. 

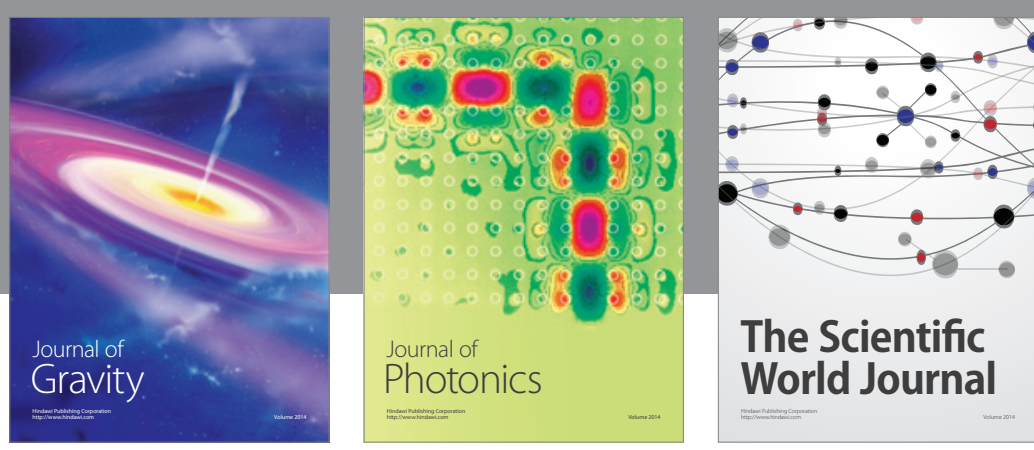

The Scientific World Journal
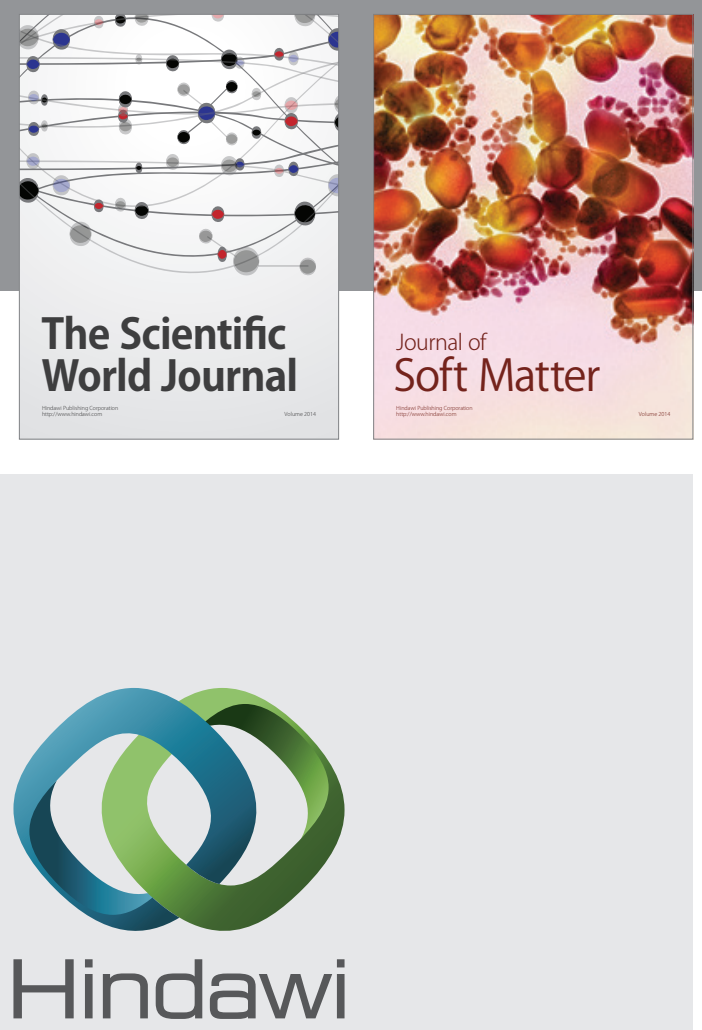

Submit your manuscripts at

http://www.hindawi.com

nternational Journal of

Statistical Mechanics
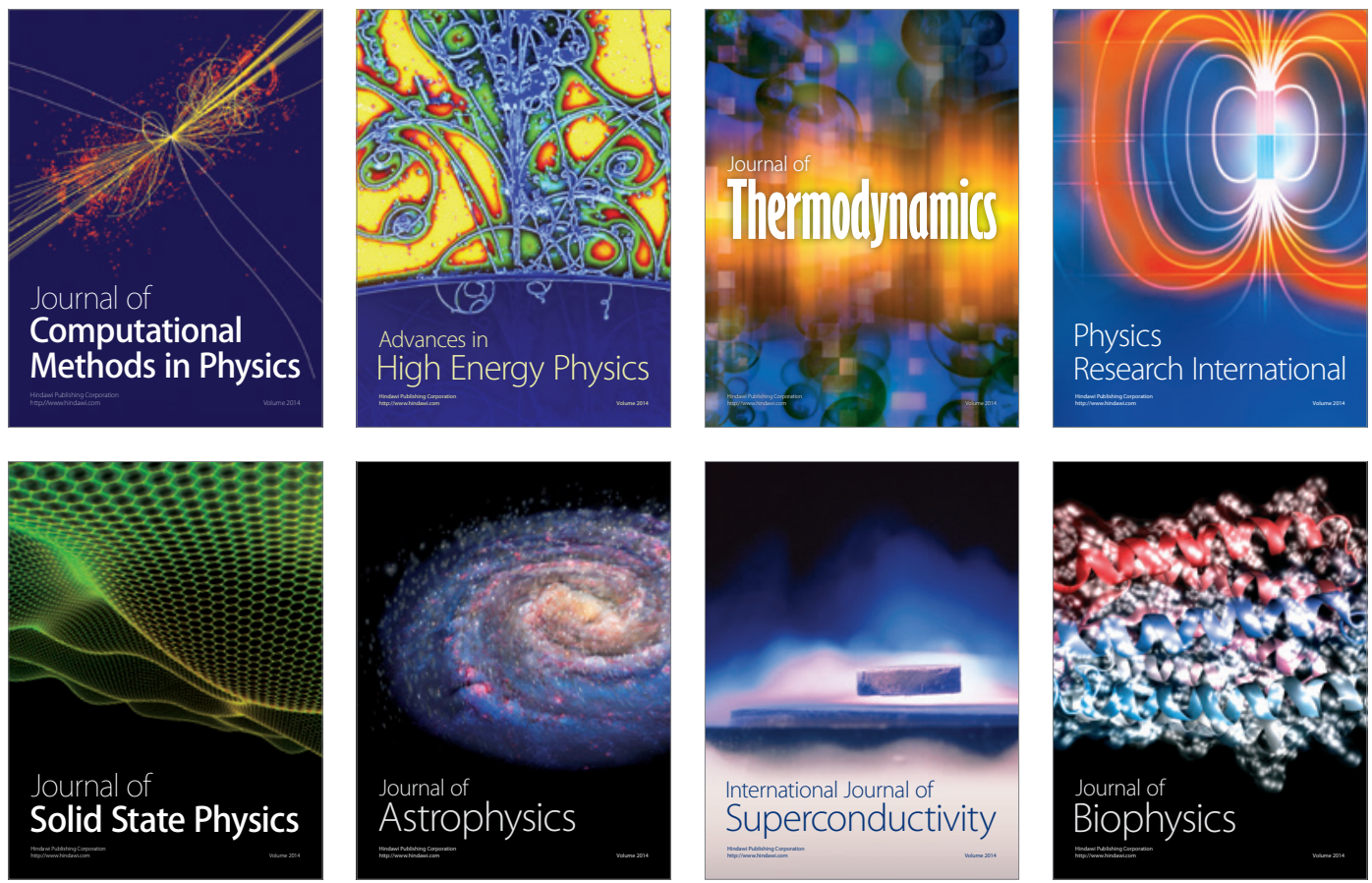
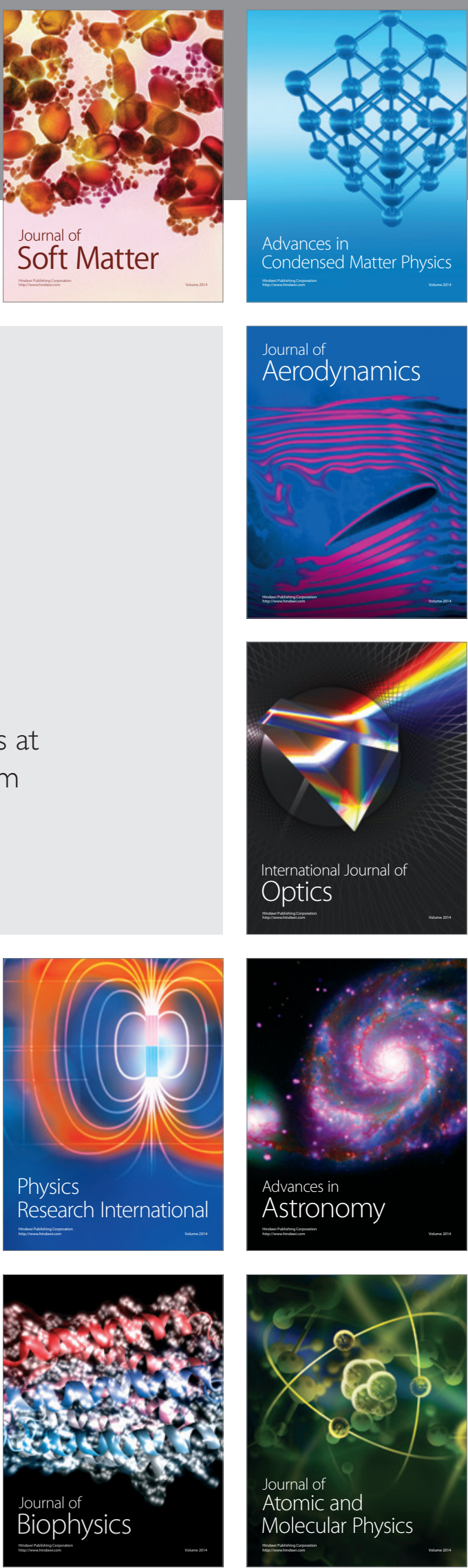